\title{
On helium-dominated stellar evolution: the mysterious role of the $\mathrm{O}(\mathrm{He})$-type stars ${ }^{\star}, \star \star$
}

\author{
N. Reindl ${ }^{1}$, T. Rauch ${ }^{1}$, K. Werner ${ }^{1}$, J. W. Kruk ${ }^{2}$, and H. Todt ${ }^{3}$ \\ ${ }^{1}$ Institute for Astronomy and Astrophysics, Kepler Center for Astro and Particle Physics, Eberhard Karls University, Sand 1, \\ 72076 Tübingen, Germany \\ e-mail: reindl@astro.uni-tuebingen.de \\ 2 NASA Goddard Space Flight Center, Greenbelt, MD 20771, USA \\ 3 Institute for Physics and Astronomy, University of Potsdam, Karl-Liebknecht-Str. 24/25, 14476 Potsdam, Germany
}

Received 23 January 2014 / Accepted 28 April 2014

\begin{abstract}
Context. About a quarter of all post-asymptotic giant branch (AGB) stars are hydrogen-deficient. Stellar evolutionary models explain the carbon-dominated H-deficient stars by a (very) late thermal pulse scenario where the hydrogen-rich envelope is mixed with the helium-rich intershell layer. Depending on the particular time at which the final flash occurs, the entire hydrogen envelope may be burned. In contrast, helium-dominated post-AGB stars and their evolution are not yet understood.

Aims. A small group of very hot, helium-dominated stars is formed by $\mathrm{O}(\mathrm{He})$-type stars. A precise analysis of their photospheric abundances will establish constraints to their evolution.

Methods. We performed a detailed spectral analysis of ultraviolet and optical spectra of four $\mathrm{O}(\mathrm{He})$ stars by means of state-of-the-art non-LTE model-atmosphere techniques.

Results. We determined effective temperatures, surface gravities, and the abundances of $\mathrm{H}, \mathrm{He}, \mathrm{C}, \mathrm{N}, \mathrm{O}, \mathrm{F}, \mathrm{Ne}, \mathrm{Si}, \mathrm{P}, \mathrm{S}, \mathrm{Ar}$, and $\mathrm{Fe}$. By deriving upper limits for the mass-loss rates of the $\mathrm{O}(\mathrm{He})$ stars, we found that they do not exhibit enhanced mass-loss. The comparison with evolutionary models shows that the status of the $\mathrm{O}(\mathrm{He})$ stars remains uncertain. Their abundances match predictions of a double helium white dwarf (WD) merger scenario, suggesting that they might be the progeny of the compact and of the luminous helium-rich sdO-type stars. The existence of planetary nebulae that do not show helium enrichment around every other $\mathrm{O}(\mathrm{He})$ star precludes a merger origin for these stars. These stars must have formed in a different way, for instance via enhanced mass-loss during their post-AGB evolution or a merger within a common-envelope (CE) of a CO-WD and a red giant or AGB star.

Conclusions. A helium-dominated stellar evolutionary sequence exists that may be fed by different types of mergers or CE scenarios. It appears likely that all these pass through the $\mathrm{O}(\mathrm{He})$ phase just before they become WDs.
\end{abstract}

Key words. stars: AGB and post-AGB - stars: evolution - stars: fundamental parameters - stars: abundances

\section{Introduction}

Quantitative spectral analyses of hot, post-asymptotic giant branch (AGB) stars revealed two distinct evolutionary sequences. Besides the well understood $\mathrm{H}$-rich sequence, a H-deficient sequence was discovered. It is composed of WolfRayet-type stars that evolve into PG 1159-type stars and finally might evolve into non-DA white dwarfs (WDs). While (very) late thermal pulse ((V)LTP) evolutionary models can explain the observed $\mathrm{He}, \mathrm{C}$, and $\mathrm{O}$ abundances in these stars (the typical abundance pattern for PG 1159 stars is $\mathrm{He}: \mathrm{C}: \mathrm{O}=$ $0.30-0.85: 0.15-0.60: 0.02-0.20$ by mass, Werner \& Herwig 2006) they do not reproduce the abundances in He-dominated

* Based on observations with the NASA/ESA Hubble Space Telescope, obtained at the Space Telescope Science Institute, which is operated by the Association of Universities for Research in Astronomy, Inc., under NASA contract NAS5-26666. Based on observations made with the NASA-CNES-CSA Far Ultraviolet Spectroscopic Explorer. Based on observations made with ESO Telescopes at the La Silla Paranal Observatory under programme IDs 091.D-0663, 090.D-0626.

$\star \star$ Figures 1-12, 21-23 and Table 1 are available in electronic form at http: //www . aanda.org stars, such as subdwarf O (sdO) stars, R Coronae Borealis (RCB) stars, extreme helium $(\mathrm{EHe})$ stars, and the $\mathrm{O}(\mathrm{He})$ stars.

Two evolutionary scenarios for the origin of RCB and EHe stars were suggested. They might either be formed by a final He-shell flash or be the merger product of a CO WD with a He WD (Jeffery et al. 2011). The significant Li content in the atmospheres of RCB stars supports the idea of an LTP to explain their origin. However, the relatively high inferred masses of RCB stars and their high F abundance supports a WD merger (Clayton et al. 2011). Zhang \& Jeffery (2012a) suggested that a double He-WD merger might also explain RCB and EHe stars. Recently, Kameswara Rao et al. (2013) found the RCB star DY Cen to be the first and only binary system among the RCB stars and their probable relatives. DY Cen is one of the hottest and most H-rich members of the RCB stars. Kameswara Rao et al. (2013) suggested that this system might have evolved from a common-envelope system to its current form. Therefore it may be possible that RCB stars form in various ways.

$\mathrm{RCB}$ and EHe stars are not the only He-rich stars for which a merger origin was suggested. Zhang \& Jeffery (2012b) presented the results of a double He-WD merger to explain the formation of He-rich, hot sdOs. These can be divided into three 
subgroups, one $\mathrm{C}$-rich and $\mathrm{N}$-poor, the other $\mathrm{N}$-rich and $\mathrm{C}$-poor, and the third one enriched in $\mathrm{C}$ and $\mathrm{N}$ (Hirsch 2009). In their numerical experiments, Zhang \& Jeffery (2012b) showed that in terms of $T_{\text {eff }}, \log g, \mathrm{C}$, and $\mathrm{N}$ abundances, the origin of the two sdO groups can be explained by different double He-WD merger models. Zhang \& Jeffery (2012b) distinguished between three types of mergers. In a slow-merger process the less massive He WD transfers its entire mass within a few minutes to form a disk around the primary He WD, which then accretes from it at a rate similar to the Eddington-accretion rate. The surface composition of the resulting star retains the N-rich composition of the accreted WD. In the fast-merger model, the secondary directly transfers its entire mass quickly to the primary surface, where heating up to $10^{8} \mathrm{~K}$ causes the material to expand and form a hot corona within a few minutes. The fast-merger model produces $\mathrm{C}$-rich stars, in which $\mathrm{N}$ is hardly observable. The composite merger includes both processes. In general, models with a merged mass below $0.7 M_{\odot}$ produce an N-rich star, while more massive ones produce a $\mathrm{C}$-rich star with a significant $\mathrm{N}$ content. Other origins of He-rich sdO stars are discussed as well. Because the merger of a He WD with a post-sdB star is predicted in one of the important binary channels for the formation of sdB stars (Han et al. 2002, 2003), Justham et al. (2011) proposed this formation channel for the previously unexplained He-rich sdO stars. Their models also reproduced the properties of the He-rich sdO stars in terms of $T_{\text {eff }}$ and $\log g$. However, in contrast to Zhang \& Jeffery (2012b), they did not include nuclear evolution in their calculations.

A more exotic group of He-dominated stars is formed by the $\mathrm{O}(\mathrm{He})$ stars. This spectroscopic class is defined by an almost pure He II absorption-line spectrum in the optical wavelength range (Méndez et al. 1986; Rauch et al. 1998). For more than 15 years, only the two central stars of planetary nebulae (CSPNe) K 1-27 ${ }^{1}$ (WD 0558-756) and LoTr 4, as well as HS 1522+6615 (WD 1522+662), and HS 2209+8229 (WD2209-8229) were known (Rauch et al. 1994, 1996, 1998). Recently, Werner et al. (2014) found four more of these objects, namely SDSS J 172854.34+361958.62, SDSS J 171916.97+365326.70, SDSS J 141812.50+024426.92, and SDSS J 075732.18+184329.28. They also pointed out that KPD $0005+5106$ is a pre-WD. Because of the He-rich surface composition of KPD $0005+5106$ (98\% by mass, Wassermann et al. 2010), we also consider it as an $\mathrm{O}(\mathrm{He})$ star. The evolutionary status of these objects has been studied only rarely. Rauch et al. (1998) proposed that $\mathrm{O}(\mathrm{He})$ stars might be successors of the luminous He-rich sdO stars and that because of ongoing mass loss, the low-gravity $\mathrm{O}(\mathrm{He})$ stars might envolve into PG 1159 stars. This possibility was studied by Miller Bertolami $\&$ Althaus (2006). They had to assume mass-loss rates, that were higher than predicted by radiation-driven wind theory, to turn $\mathrm{O}(\mathrm{He})$ stars into helium-enriched PG 1159 stars. An alternative scenario was suggested by Rauch et al. (2006). They invoked the idea that the $\mathrm{O}(\mathrm{He})$ stars could also be the offspring of a merging event of two WDs and thus, the direct descendants of RCB stars.

In this paper, we present the analysis of K 1-27, LoTr 4, HS 1522+6615, and HS 2209+8229. In Sect. 2, we briefly describe the available observations. We then present a detailed spectral analysis of the $\mathrm{O}(\mathrm{He})$ stars based on optical and ultraviolet spectra (Sect. 3) and derive stellar parameters and distances (Sect. 4). In Sect. 5 we discuss possible evolutionary channels for the $\mathrm{O}(\mathrm{He})$ stars and those of other helium-dominated objects. We conclude in Sect. 5.3.

1 In the following, we use the PNe names to identify their central stars.

\section{Observations}

In January, June and July 2013, we performed observations of K 1-27 and LoTr 4 with the ESO/NTT (ProgIDs 091.D-0663, 090.D-0626) using the EFOSC2 spectrograph (resolving power $R=\lambda / \Delta \lambda \approx 3000$ ) with the grisms GR\#19 (4400 to $5100 \AA)$ and GR\#20 (6000 to $7100 \AA$ ). The data reduction was done by using $\mathrm{IRAF}^{2}$. The previous optical spectra of the $\mathrm{O}(\mathrm{He})$ stars were already described and analyzed by Rauch et al. (1994, 1996, $1998)$ to determine effective temperature $\left(T_{\text {eff }}\right)$, surface gravity $(\log g)$, and the $\mathrm{H} / \mathrm{He}$ ratio for all four stars as well as the $\mathrm{C}$ abundance for K 1-27, LoTr 4 (upper limits), HS 1522+6615, and the $\mathrm{N}$ abundance for K 1-27 and LoTr 4.

We have taken FUV spectra with $\operatorname{FUSE}^{3}(R \approx 20000)$ in 2002 (all $\mathrm{O}(\mathrm{He})$ stars, $49 \mathrm{ks}$ exposure time in total, ProgID: C178) and 2005 (only HS 1522+6615, 4 ks, ProgID: U103) using the LWRS aperture (Figs. 1-4). Additional $204 \mathrm{ks}$ of FUSE observations (K 1-27, HS 1522+6615, HS 2209+8229) were scheduled for summer 2007, but were not performed because of the FUSE failure on July 12. The FUSE spectra show a strong contamination by interstellar (IS) line absorption and the $\mathrm{S} / \mathrm{N}$ ratio is very poor (Rauch et al. 2006, 2009). To reduce the pixel-to-pixel variation, they were co-added and then slightly smoothed with a low-pass filter (Savitzky \& Golay 1964).

Because of the poor quality of the FUSE spectra, we obtained HST/COS ${ }^{4}$ spectra (Proposal Id: 11699, Figs. 5-8) during April to July 2010 using the grating G140L $(2000<R<3500$ within $1150 \AA<\lambda<1800 \AA$ ) and the primary science aperture.

\section{Spectral analysis}

Motivated by the new COS spectra, we decided to comprehensively re-analyze all available data of the $\mathrm{O}(\mathrm{He})$ stars. Since the time when Rauch et al. (1998) presented their analysis of optical, ultraviolet $\left(\mathrm{IUE}^{5}\right)$, and X-ray $\left(\mathrm{ROSAT}^{6}\right)$ data, our Tübingen NLTE Model Atmosphere Package TMAP ${ }^{7}$ (Sect. 3.1) as well as the atomic data, that is taken from $\mathrm{TMAD}^{8}$, the Tübingen Model Atom Database, have continuously improved. Moreover, in a standard procedure, we modeled photospheric and interstellar line-absorption spectra to correctly identify the pure atmospheric lines. Absorption by interstellar gas was modeled using the program Owens (Hébrard \& Moos 2003; Hébrard et al. 2002). Owens allows for multiple independent interstellar clouds, each with its own radial and turbulent velocities, temperature, and column densities for each of any number of constituent gas species. All properties of each cloud are adjusted by fitting Voigt profiles to the data by chi-squared minimization.

\subsection{Model atmospheres}

We used TMAP (Werner et al. 2003; Rauch \& Deetjen 2003) to compute plane-parallel, line-blanketed non-LTE model atmospheres in radiative and hydrostatic equilibrium. In the previous

\footnotetext{
2 IRAF is distributed by the National Optical Astronomy Observatory, which is operated by the Associated Universities for Research in Astronomy, Inc., under cooperative agreement with the National Science Foundation.

3 Far Ultraviolet Spectroscopic Explorer.

4 Cosmic Origins Spectrograph.

International Ultraviolet Explorer.

6 Röntgensatellit.

http://astro.uni-tuebingen.de/ TMAP

8 http://astro.uni-tuebingen.de/ TMAD
} 


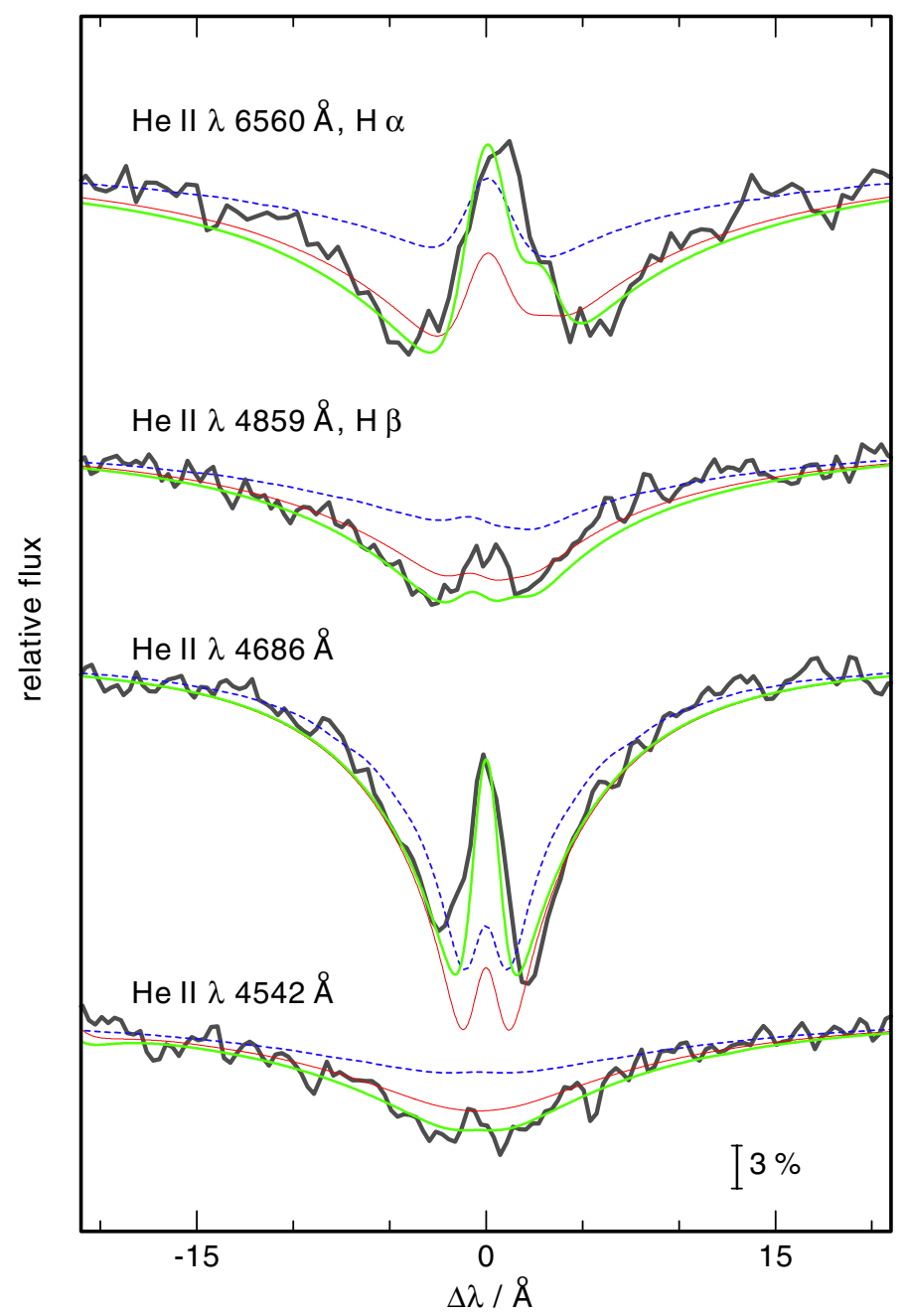

Fig. 13. Comparison of optical $\mathrm{H}$ I and He II lines calculated from models including $\mathrm{H}+\mathrm{He}$ (dashed, blue), $\mathrm{H}+\mathrm{He}+\mathrm{C}+\mathrm{N}+\mathrm{O}$ (thin, red), and $\mathrm{H}+\mathrm{He}+\mathrm{C}+\mathrm{N}+\mathrm{O}+\mathrm{Ne}$ (thick, green) with $T_{\text {eff }}=135 \mathrm{kK}$ and $\log g=6.4$, overplotted on the EFOSC2 spectrum of K 1-27. The vertical bar indicates $3 \%$ of the continuum flux.

analysis of the O(He) stars, Rauch et al. (1994, 1996, 1998) used only $\mathrm{H}+\mathrm{He}$ model atmospheres to derive $T_{\text {eff }}, \log g$, and the $\mathrm{H} / \mathrm{He}$ ratio. In our analysis, we also included the elements $\mathrm{C}, \mathrm{N}$, $\mathrm{O}, \mathrm{F}, \mathrm{Ne}, \mathrm{Si}, \mathrm{P}, \mathrm{S}$, and $\mathrm{Ar}$ with their dominant ionization stages (Table 1, Figs. 9-12) to study their impact on the model atmospheres and the resulting line profiles. We found that $\mathrm{C}, \mathrm{N}$, $\mathrm{O}$, and $\mathrm{Ne}$ have a strong influence on the atmospheric structure. In Fig. 13 we demonstrate the strong effect of additional opacities on the resulting line profiles. The impact of F, Si, P, S, and Ar turned out to be negligible. Dreizler \& Werner (1994) have shown that line blanketing by iron-group elements hardly affects the hydrogen and helium lines in hot central stars. To compute a model grid in reasonable time, we therefore decided to include only $\mathrm{H}, \mathrm{He}, \mathrm{C}, \mathrm{N}, \mathrm{O}$, and $\mathrm{Ne}$ in our model atmospheres to derive $T_{\text {eff }}, \log g$, and the element abundances of these elements. To determine the abundances of F, Si, P, S, Ar, and Fe, we kept the values of $T_{\text {eff }}$ and $\log g$ fixed. The upper limits (Table 3 ) were derived by test models where the respective lines in the model contradict the non-detection of the lines in the observation (at the abundance limit).

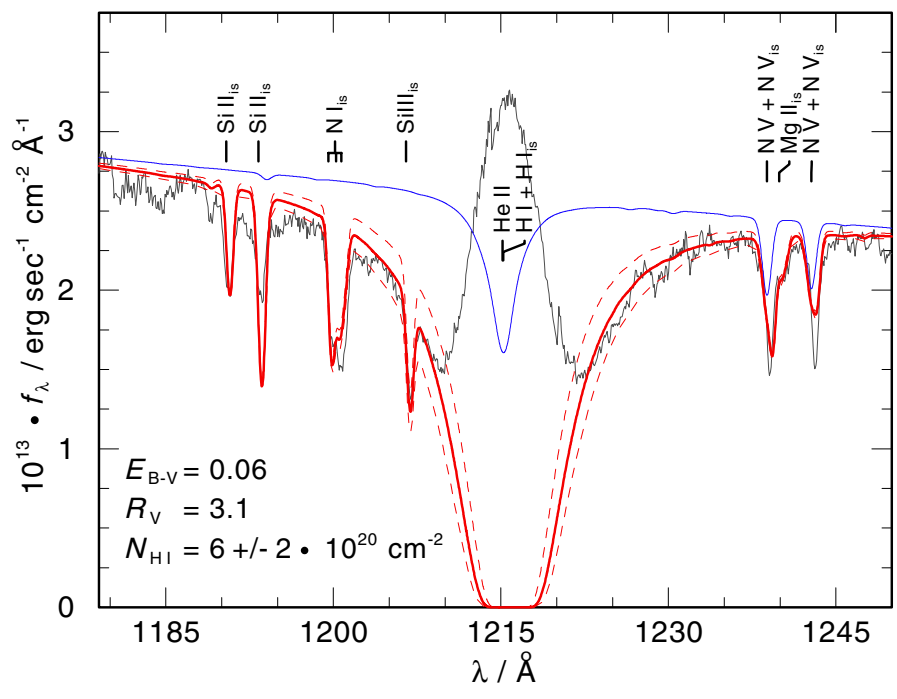

Fig. 14. Determination of the interstellar H I column density toward K 1-27. The dashed lines indicate the error limits. The thin (blue) line is the pure photospheric spectrum. Prominent spectral lines are marked at top, "is" indicates interstellar origin. The line center of Ly $\alpha$ is dominated by geocoronal emission.

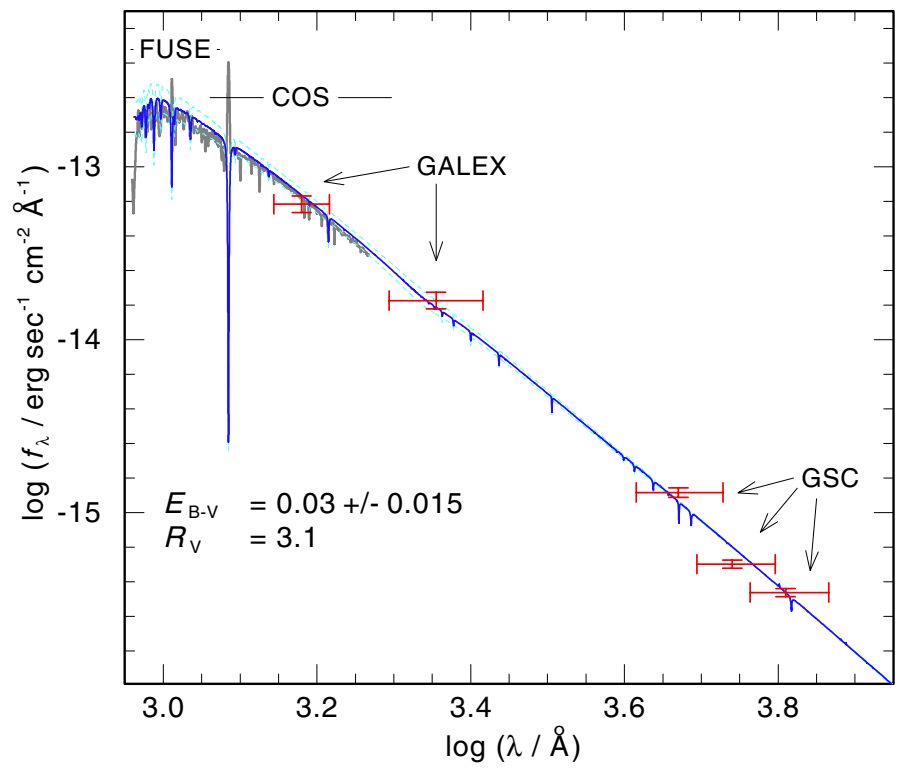

Fig. 15. Determination of $E_{B-V}$ for HS $1522+6615$. The FUSE and COS spectra and GALEX and BVR brightnesses from the GSC 2.3 catalog are used for comparison. The thick (blue) line is the spectrum of the final photospheric model. The dashed lines indicate the error limits.

\subsection{Interstellar neutral hydrogen and reddening}

The interstellar H I column density was measured from Ly $\alpha$ (Fig. 14). Interstellar reddening was determined using the reddening law of Fitzpatrick (1999). Since its impact is negligible in the infrared, the model flux was normalized to the most reddest brightness found in the literature. Figure 15 shows the example of HS $1522+6615$, where the $\mathrm{GSC}^{9} \mathrm{R}$ brightness is used. Table 2 summarizes $N_{\mathrm{HI}}$ and $E_{B-V}$ of the $\mathrm{O}(\mathrm{He})$ stars compared with GALEX ${ }^{10}$ values, which agree well. Within the error limits, our values agree with those of Rauch et al. (1998).

\footnotetext{
9 Guide Star Catalogue, Bucciarelli et al. (2001).

${ }^{10}$ Galaxy Evolution Explorer, http://www.galex.caltech.edu/
} 
Table 2. Interstellar H I column density and reddening.

\begin{tabular}{cccc}
\hline \hline & & \multicolumn{2}{c}{$E_{B-V}$} \\
\cline { 3 - 4 } Object & $N_{\mathrm{HI}}\left[10^{20} \mathrm{~cm}^{-2}\right]$ & this work & GALEX \\
\hline K 1-27 & $6.0 \pm 2.0$ & $0.06 \pm 0.020$ & 0.0797 \\
LoTr 4 & $10.0 \pm 1.5$ & $0.20 \pm 0.020$ & 0.1893 \\
HS 1522+6615 & $2.0 \pm 1.0$ & $0.03 \pm 0.015$ & 0.0250 \\
HS 2209+8229 & $5.0 \pm 1.0$ & $0.23 \pm 0.040$ & \\
\hline
\end{tabular}

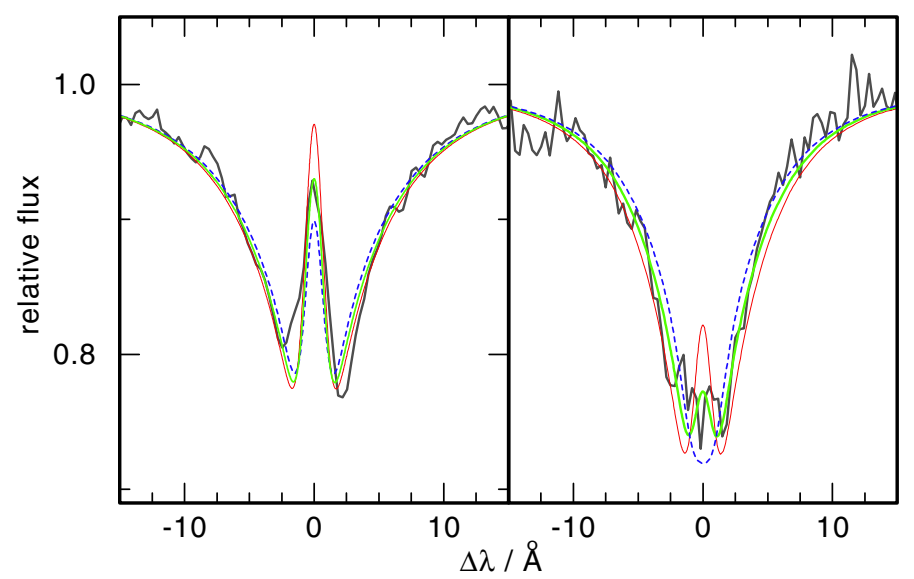

Fig. 16. Determination of $T_{\text {eff }}$ of K 1-27 (left) and LoTr 4 (right) using the central emission of He II $\lambda 4686 \AA$. The EFOSC 2 observation (solid black line) is compared with models with $T_{\text {eff }}=130 / 110 \mathrm{kK}$ (dashed, blue), 135/120 kK (thick, green), and 140/130 kK (thin, red).

\subsection{Effective temperature and surface gravity}

To determine of $T_{\text {eff }}$ and $\log g$, we used the optical H I and He II lines. For HS $1522+6615$ and HS 2209+8229 we additionally used the $\mathrm{O}$ ionization equilibria to constrain $T_{\text {eff }}$. The new optical observations of K 1-27 and LoTr 4 allowed us to use the central emission of He II $\lambda 4686 \AA$ to derive $T_{\text {eff }}$ for the two CSPNe (Fig. 16).

For K $1-27, T_{\text {eff }}=135 \mathrm{kK}$ and $\log g=6.4(\mathrm{~cm} / \mathrm{s})$ were found. The value of $\log g$ agrees with Rauch et al. (1994, 1998, $\log g=6.5 \pm 0.5)$, but for $T_{\text {eff }}$ we found a large deviation from the result of Rauch et al. $\left(1998, T_{\text {eff }}=105 \pm 10 \mathrm{kK}\right)$. However, Rauch et al. (1994, 1998) encountered problems with the lower value of $T_{\text {eff }}$ which can be solved with $T_{\text {eff }}=135 \mathrm{kK}$ : first, with $T_{\text {eff }}=105 \mathrm{kK}$ the star did not provide enough hard photons to ionize its nebula. With the higher $T_{\text {eff }}$ for $\mathrm{K} 1-27$, we achieve a more consistent PN $\leftrightarrow$ CSPN model. Second, $\mathrm{N} v \lambda \lambda 4604,4620 \AA$ do not appear in emission at $T_{\text {eff }}=105 \mathrm{kK}$ (as the observation shows), but for $T_{\text {eff }}=135 \mathrm{kK}$ they do. Finally, Rauch et al. (1994) had the problem that the central depression of the He II $\lambda 4686 \AA\left(n-n^{\prime}=3-4\right)$ line was too strong, while other $\mathrm{HI}$ and He II lines were almost perfectly reproduced. The addition of $\mathrm{Ne}$ to the models solves this problem. Furthermore, the central He II $\lambda 4686 \AA$ and $\mathrm{H} \alpha$ emission can only be reproduced with a supersolar Ne abundance, which causes a strong decrease of the temperature in the outer atmosphere. He II $\lambda 4686 \AA$ and $\mathrm{H} \alpha$ are most affected by this because they are formed at lower Rosseland optical depths. We stress that the large deviation from the previous $T_{\text {eff }}$ value is a result of the additional opacities used in our model atmospheres, as mentioned above.

For LoTr 4, we confirmed the value of $T_{\text {eff }}=120 \mathrm{kK}$ (Rauch et al. 1996) but increased the lower limit to $T_{\text {eff }}=115 \mathrm{kK}$ because for lower values, N v $\lambda \lambda 4604,4620 \AA$ do not appear in

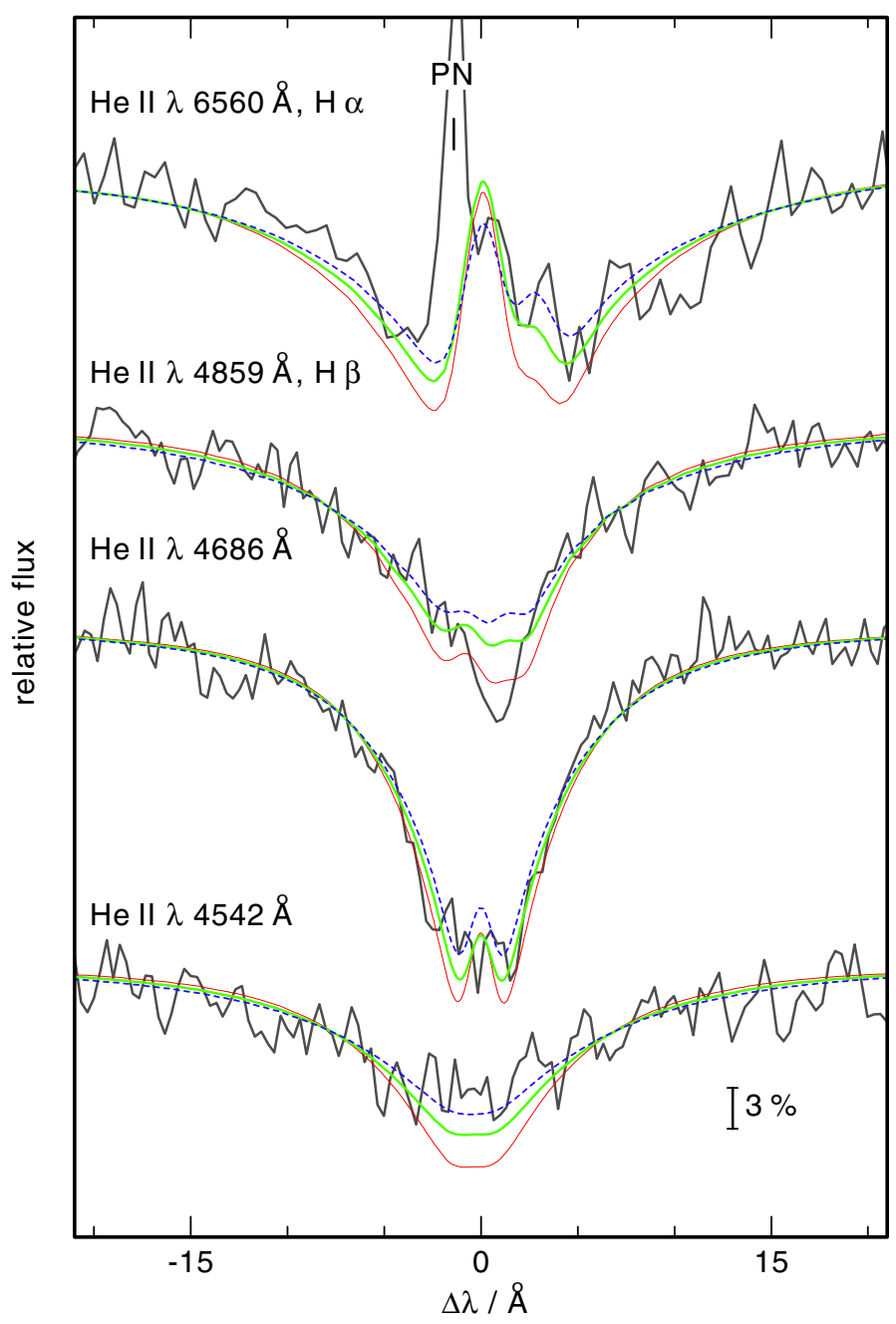

Fig. 17. Determination of $\log g$ of LoTr 4. The EFOSC2 observation (solid black line) is compared with models with $\log g=6.0$ (dashed, blue), 5.8 (thick, green), and 5.6 (thin, red). The vertical bar indicates $3 \%$ of the continuum flux.

emission as in the observation. The new optical spectra allowed us a better determination of the surface gravity (Fig. 17). We found $\log g=5.8 \pm 0.2$, which is within the error limits of the literature value (Rauch et al. 1996, $\log g=5.5 \pm 0.5$ ).

For HS $1522+6615$, we determined a lower value of $T_{\text {eff }}=130 \mathrm{kK}$ at a higher $\log g=5.9$ (compared with Rauch et al. $\left.1998, T_{\text {eff }}=140 \pm 10 \mathrm{kK}, \log g=5.5 \pm 0.5\right)$. The errors of $\log g$ were reduced to \pm 0.2 .

Based on the O IV/O v ionization equilibrium (Fig. 18), we found a higher $T_{\mathrm{eff}}=110 \mathrm{kK}$ compared with the literature value $\left(T_{\text {eff }}=100 \pm 10 \mathrm{kK}\right)$ for HS 2209+8229. The value of $\log g=6.0$ is verified and the errors were reduced to \pm 0.3 .

\subsection{Element abundances}

The $\mathrm{H} / \mathrm{He}$ abundance ratio (by mass) was determined using H I $\lambda \lambda 6563,4861,4340 \AA$ and He II $\lambda \lambda 6560,5412,4859$, $4686,4542,4339 \AA$. It was difficult to fix because the quality of the available optical observations is poor. For LoTr 4 and $\mathrm{K} 1-27$, we reverified the literature values of 0.5 and 0.2 , respectively. Figure 19 shows that for HS $1522+6615$ the model with $\mathrm{H} / \mathrm{He}=0.03$ fits the observation better than $\mathrm{H} / \mathrm{He}=0.10$ (literature value) where $\mathrm{H} \alpha$ appears in emission while for $\mathrm{H} / \mathrm{He}=0.01$ 
N. Reindl et al.: On helium-dominated stellar evolution: the mysterious role of the $\mathrm{O}(\mathrm{He})$-type stars

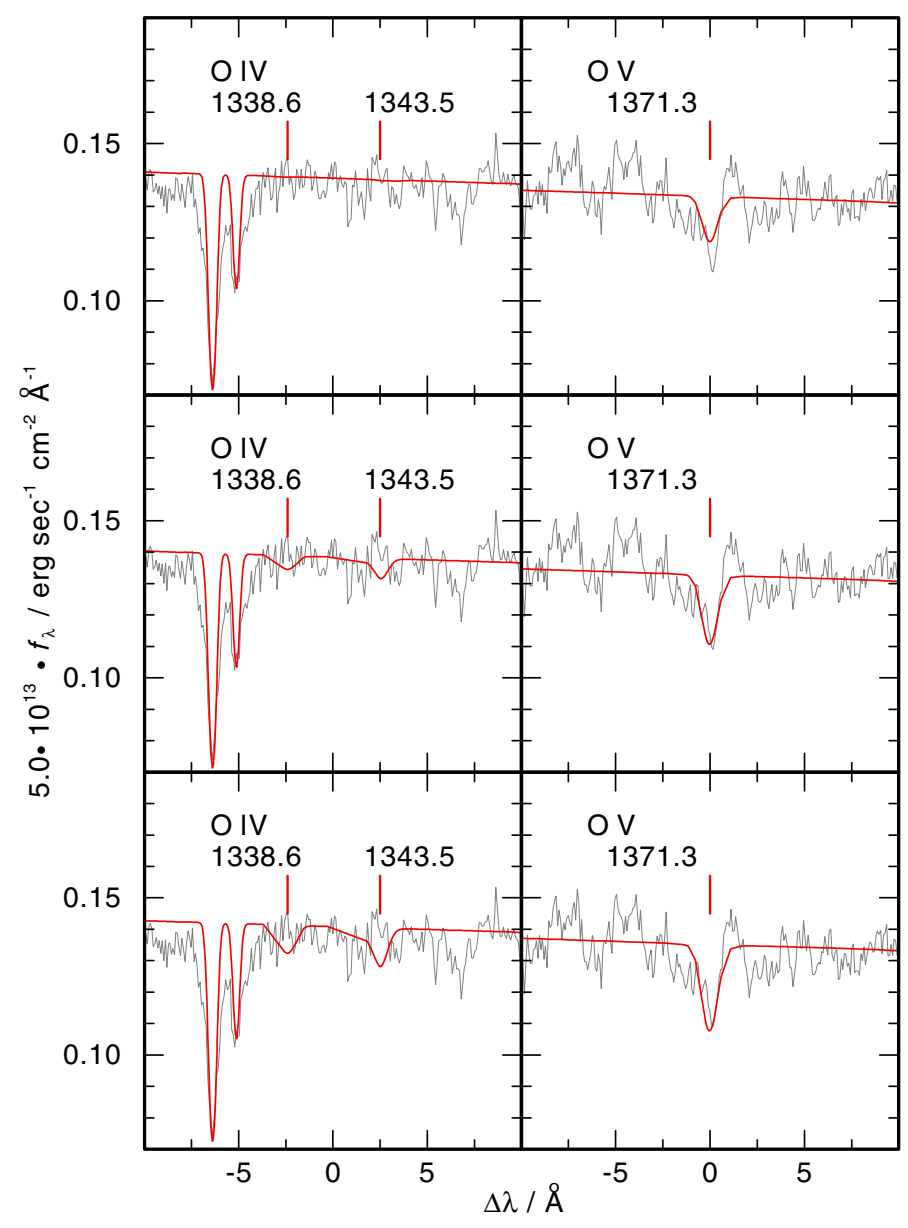

Fig. 18. Determination of $T_{\text {eff }}$ of HS 2209+8229 from the O IV/O V ionization equilibrium. Top panels: $T_{\mathrm{eff}}=100 \mathrm{kK}$, middle: $T_{\mathrm{eff}}=110 \mathrm{kK}$; bottom: $T_{\mathrm{eff}}=120 \mathrm{kK}$.

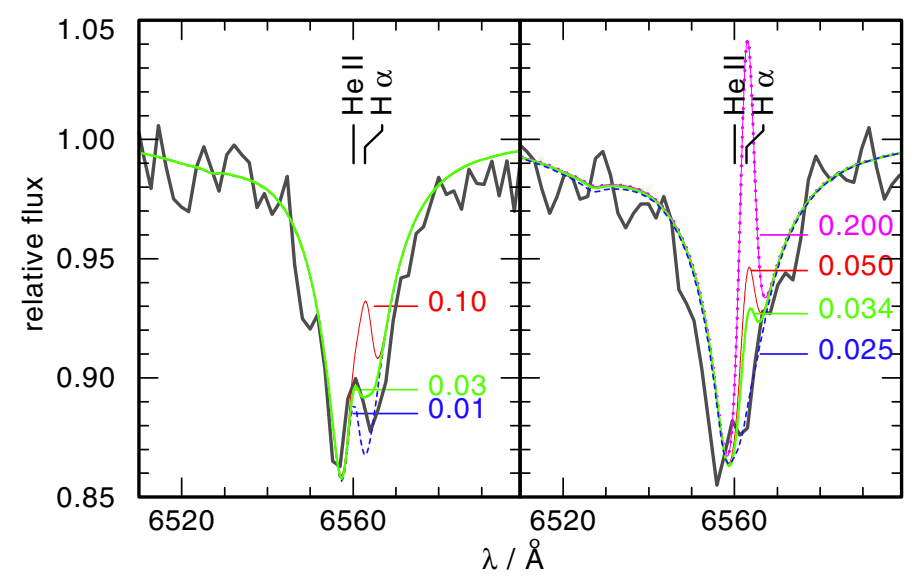

Fig. 19. Determination of the $\mathrm{H} / \mathrm{He}$ ratio of HS $1522+6615$ (left panel) and HS 2209+8229 (right). The observed $\mathrm{H} \alpha$ and He II line blend is compared with models with different $\mathrm{H} / \mathrm{He}$ mass ratios, as indicated by the labels.

$\mathrm{H} \alpha$ is too deep in absorption. For HS 2209+8229, we found that the upper limit of the $\mathrm{H} / \mathrm{He}$ ratio must be lower because of the strong $\mathrm{H} \alpha$ emission for $\mathrm{H} / \mathrm{He}>0.025$ (Table 3, Fig. 20).

Carbon. The C IV $\lambda \lambda 1548.2,1550.8 \AA$ resonance doublet in the COS spectra of all four stars is contaminated by the respective interstellar lines and therefore unsuited for determining the
C abundance. In the spectra of LoTr 4 and HS 2209+8229 no other $\mathrm{C}$ lines were identified and we derived only upper limits of $[\mathrm{C}]^{11}<-0.53$ and $[\mathrm{C}]<-1.50$, respectively. In the spectrum of K 1-27, we identified C IV $\lambda \lambda 1168.8,1168.9 \AA$ and determined $[\mathrm{C}]=-0.62$. For HS $1522+6615$, C IV $\lambda \lambda 1168.9,1169.0 \AA$ in the COS spectrum and CIV $\lambda \lambda 5801.33,5811.98 \AA$ in the optical spectrum were evaluated, and we achieve the best agreement between model and observation at $[\mathrm{C}]=0.62$. HS $1522+6615$ shows the highest, supersolar $C$ abundance (Fig. 21) while the upper limits of the other stars in our sample indicate subsolar abundances.

Nitrogen. The N abundance for K 1-27 and LoTr 4 was determined from the N v $\lambda \lambda 4603.7,4619.7,4933.6,4943.2,4945.7 \AA$ emission lines. For HS 2209+8229, we used N v $\lambda \lambda 1616.1 \AA$, $1616.4,1619.6,1619.7$. As for case of C IV, the resonance doublet $\mathrm{N}$ V $\lambda \lambda 1238.8,1242.8 \AA$ is blended by the respective ISM lines. The $\mathrm{N}$ abundances of K $1-27([\mathrm{~N}]=1.28$, Fig. 22), LoTr 4 $([\mathrm{N}]=1.07)$, and HS 2209+8229 $([\mathrm{N}]=2.89)$ were found to be supersolar, in contrast to the upper limit for HS $1522+6615$ $([\mathrm{N}]<-0.78)$, which is subsolar.

Oxygen. For K 1-27 and LoTr4, we derived only upper limits because no $\mathrm{O}$ lines except the resonance doublet O VI $\lambda \lambda 1031.9,1037.7 \AA$ were identified, which might be of ISM origin similar to the $\mathrm{CIV}$ and $\mathrm{NV}$ resonance doublets (Sect. 3.4) We used the OVI $\lambda \lambda 1124.7,1124.8 \AA$ and O VI $\lambda 5290.6 \AA$ to determine the upper limits of [O] $<-2.06$ for $\mathrm{K} 1-27$ and $[\mathrm{O}]<-1.47$ for LoTr 4 . For HS 1522+6615, Rauch et al. (1998) discovered a variability of the O VI $\lambda 5290.6 \AA$ emission feature. Mickaelian et al. (2011) also classified HS $1522+6615$ as "possible" variable by comparing the brightness measurements from the Palomar Observatory Sky Survey (POSS) epochs 1 and 2. In view of its variability, we adjusted the $\mathrm{O}$ abundance to the co-added optical spectra using O VI $\lambda \lambda 3811.4,3834.2 \AA$ and found [O] $=-0.64$. A precise determination of the $\mathrm{O}$ abundance was possible for HS 2209+8229 using O v $\lambda 1371.3 \AA$, which is prominent in the COS spectrum (Fig. 23). We found [O] $=-1.46$ (Table 3 ).

Fluorine. $\mathrm{F}$ is not identified in the $\mathrm{O}(\mathrm{He})$ star spectra. We used F VI $\lambda 1139.5 \AA$ and found $[\mathrm{F}]<-1.00$ for all $\mathrm{O}(\mathrm{He})$ stars.

Neon. We only derived upper limits using Ne VII $\lambda \lambda$ 1438.8, $1440.5,1445.0 \AA$ for K 1-27, LoTr 4, and HS 1522+6615 and Ne V $\lambda \lambda 1718.2,1718.3 \AA$ for HS $2209+8229$ because of its lower $T_{\text {eff. }}$ We determined $[\mathrm{Ne}]<0.60$ for $\mathrm{K} 1-27,[\mathrm{Ne}]<$ -0.09 for LoTr $4,[\mathrm{Ne}]<-0.62$ for HS $1522+6615$, and $[\mathrm{Ne}]<$ -1.10 for HS $2209+8229$.

Silicon. An upper limit for the Si abundance was determined using Si IV $\lambda 1128.3 \AA$, Si V $\lambda 1118.8 \AA$, and Si VI $\lambda 1130.4 \AA$. For $\mathrm{K} 1-27$, it is [Si] < -0.56. For LoTr 4, HS 1522+6615, and HS 2209+8229, we derived [Si] $<0.18$.

Phosphorus. We identified P v $\lambda \lambda 1118.0,1128.0 \AA$ only in the FUSE spectra of LoTr 4 and HS 2209+8229, and we determined

11 [X] denotes $\log$ (abundance by mass/solar abundance by mass) of species X. Solar abundances were taken from Asplund et al. (2009). 
Table 3. Photospheric parameters of the $\mathrm{O}(\mathrm{He})$ stars.

\begin{tabular}{|c|c|c|c|c|c|c|c|c|}
\hline & \multicolumn{2}{|c|}{ K 1-27 } & \multicolumn{2}{|c|}{ LoTr 4} & \multicolumn{2}{|c|}{ HS $1522+6615$} & \multicolumn{2}{|c|}{ HS $2209+8229$} \\
\hline & R14 & R98 & R14 & R98 & R14 & R98 & R14 & R98 \\
\hline $\begin{array}{r}T_{\text {eff }} \\
\mathrm{kK}\end{array}$ & $135_{-5}^{+5}$ & $105_{-10}^{+10}$ & $120_{-5}^{+10}$ & $120_{-12}^{+12}$ & $130_{-10}^{+10}$ & $140_{-14}^{+14}$ & $110_{-10}^{+10}$ & $100_{-10}^{+10}$ \\
\hline $\begin{array}{r}\log g \\
\mathrm{~cm} \mathrm{~s}^{-2}\end{array}$ & $6.4_{-0.3}^{+0.2}$ & $6.5_{-0.5}^{+0.5}$ & $5.8_{-0.2}^{+0.2}$ & $5.5_{-0.5}^{+0.5}$ & $5.9_{-0.2}^{+0.2}$ & $5.5_{-0.5}^{+0.5}$ & $6.0_{-0.3}^{+0.3}$ & $6.0_{-0.5}^{+0.5}$ \\
\hline $\mathrm{H}$ & $-1.33_{-0.28}^{+0.41}$ & $<-1.30$ & $-0.92_{-0.20}^{+0.21}$ & $-0.90_{-0.30}^{+0.30}$ & $-2.08_{-0.52}^{+0.47}$ & $-1.60_{-0.30}^{+0.30}$ & $<-2.21$ & $<-1.30$ \\
\hline $\mathrm{He}$ & $-0.03_{-0.03}^{+0.03}$ & $>-0.02$ & $-0.06_{-0.05}^{+0.02}$ & $-0.06_{-0.3}^{+0.3}$ & $-0.009_{-0.009}^{+0.005}$ & $0.004_{-0.3}^{+0.3}$ & $>-0.004$ & $>-0.02$ \\
\hline $\mathrm{C}$ & $-3.25_{-0.55}^{+0.45}$ & $<-1.82$ & $<-3.14$ & $<-1.92$ & $-2.00_{-0.30}^{+0.68}$ & $-2.05_{-0.3}^{+0.3}$ & $<-4.13$ & \\
\hline $\mathrm{N}$ & $-1.88_{-0.42}^{+0.36}$ & $-1.76_{-0.3}^{+0.3}$ & $-2.10_{-0.92}^{+0.60}$ & $-2.45_{-0.3}^{+0.3}$ & $<-3.94$ & & $-2.89_{-0.50}^{+0.89}$ & \\
\hline $\mathrm{O}$ & $<-4.30$ & & $<-3.73$ & $<-1.50$ & $-2.88_{-0.50}^{+0.60}$ & & $-3.00_{-0.70}^{+0.70}$ & \\
\hline $\mathrm{F}$ & $<-7.30$ & & $<-7.29$ & & $<-7.30$ & & $<-7.45$ & \\
\hline $\mathrm{Ne}$ & $<-2.30$ & & $<-2.99$ & & $<-3.53$ & & $<-4.00$ & \\
\hline $\mathrm{Si}$ & $<-4.06$ & & $<-3.00$ & & $<-3.00$ & & $<-3.00$ & \\
\hline $\mathrm{P}$ & $<-5.52$ & & $-4.54_{-1.00}^{+0.40}$ & & $<-5.20$ & & $-6.00_{-1.00}^{+1.00}$ & \\
\hline $\mathrm{S}$ & $<-4.07$ & & $-3.43_{-1.00}^{+0.61}$ & & $<-3.59$ & & $-3.40_{-1.27}^{+0.88}$ & \\
\hline $\mathrm{Ar}$ & $<-5.30$ & & $<-4.92$ & & $<-5.10$ & & $<-6.26$ & \\
\hline $\mathrm{Fe}$ & $<-2.89$ & & $<-1.89$ & & $<-2.89$ & & $<-2.89$ & \\
\hline
\end{tabular}

Notes. Our values (R14) are compared with those given by Rauch et al. (1998, R98). Abundances are given as logarithmic mass fraction.

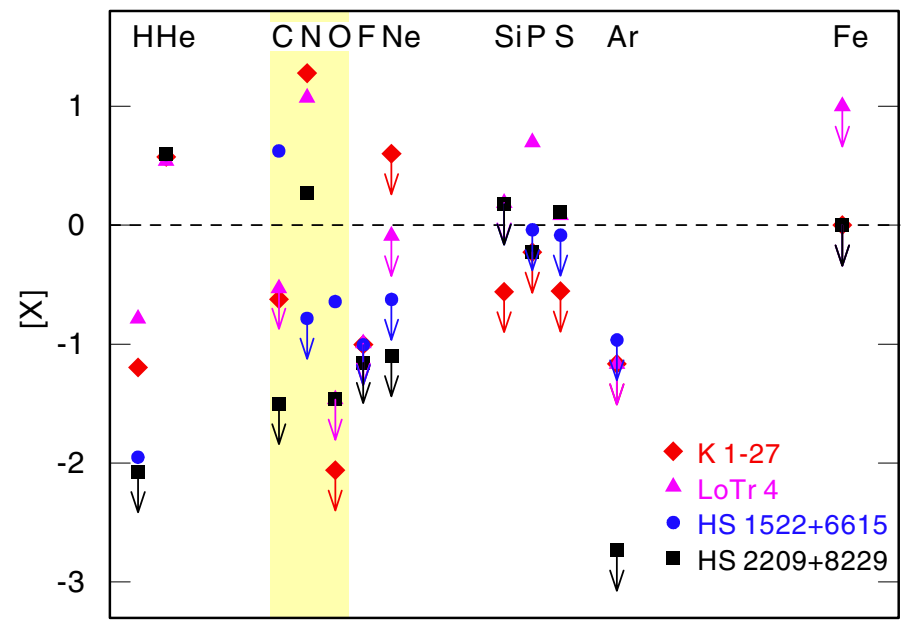

Fig. 20. Abundances of the $\mathrm{O}(\mathrm{He})$ stars (Table 3 ). The shaded (yellow) region emphasizes the $\mathrm{CNO}$ differences.

$[\mathrm{P}]=0.70$ and $[\mathrm{P}]=-0.76$, respectively. For $\mathrm{K} 1-27$ and HS $1522+6615$, only upper limits can be given, which are about solar.

Sulfur. S VI $\lambda 1117.8 \AA$ was identified in the FUSE spectra of LoTr 4 and HS 2209+8229 and we determined $[S]=-0.08$ and $[\mathrm{S}]=-0.11$, respectively. For K 1-27 and HS 1522+6615, only upper limits can be given, which are 0.3 times solar and solar, respectively.

Argon. Is not identified in the $\mathrm{O}(\mathrm{He})$ star spectra. We employed Ar VII $\lambda 1535.7,1537.1,1537.9 \AA$ and Ar VIII $\lambda 1164.1 \AA$ and derived subsolar upper limits.
Iron. The quality of the FUSE spectra is not sufficient to identify individual Fe lines. Therefore, we only included the iron model atom (ionization stages V - IX) in the line-formation calculations (i.e. fixed atmospheric structure) based on our final model and calculated NLTE occupation numbers for the atomic levels of iron. We found that the resulting upper limits of the Fe abundances are solar for K 1-27, HS 1522+6615, and HS 2209+8229. For LoTr 4, we found an upper limit of ten times solar.

\subsection{Mass-loss rates}

One aim of our COS observations was to follow up on the suggestion of Miller Bertolami \& Althaus (2006) that $\mathrm{O}(\mathrm{He})$ stars might be post early-AGB stars. The high mass-loss rates invoked in their calculations were not confirmed by our FUSE observations. We performed test calculations based on the final parameters of our analysis (Table 3) using the Potsdam Wolf-Rayet model-atmosphere code, PoWR ${ }^{12}$. This code solves the NLTE radiative transfer in a spherically expanding atmosphere simultaneously with the statistical equilibrium equations and accounts at the same time for energy conservation. Like in TMAP, irongroup line blanking is treated by means of the superlevel approach (Gräfener et al. 2002), and a wind clumping in first-order approximation is taken into account (Hamann \& Gräfener 2004). We did not calculate hydrodynamically consistent models, but assumed a velocity field following a $\beta$-law with $\beta=1$.

For our calculations, we first used the predicted mass-loss rates $(\dot{M})$ of Pauldrach et al. (1988, Table 4) using the stellar masses determined from comparison with evolutionary tracks of VLTP stars (Fig. 24, Miller Bertolami \& Althaus 2006). We considered the terminal wind velocities $\left(v_{\infty}\right)$ to be 2.51 times the escape velocities of the stars (Lamers et al. 1995). To then determine an upper limit we increased the mass-loss rate until the synthetic spectra no longer agreed with the observations. We

12 http://www . astro.physik. uni-potsdam.de/ wrh/PoWR/ 


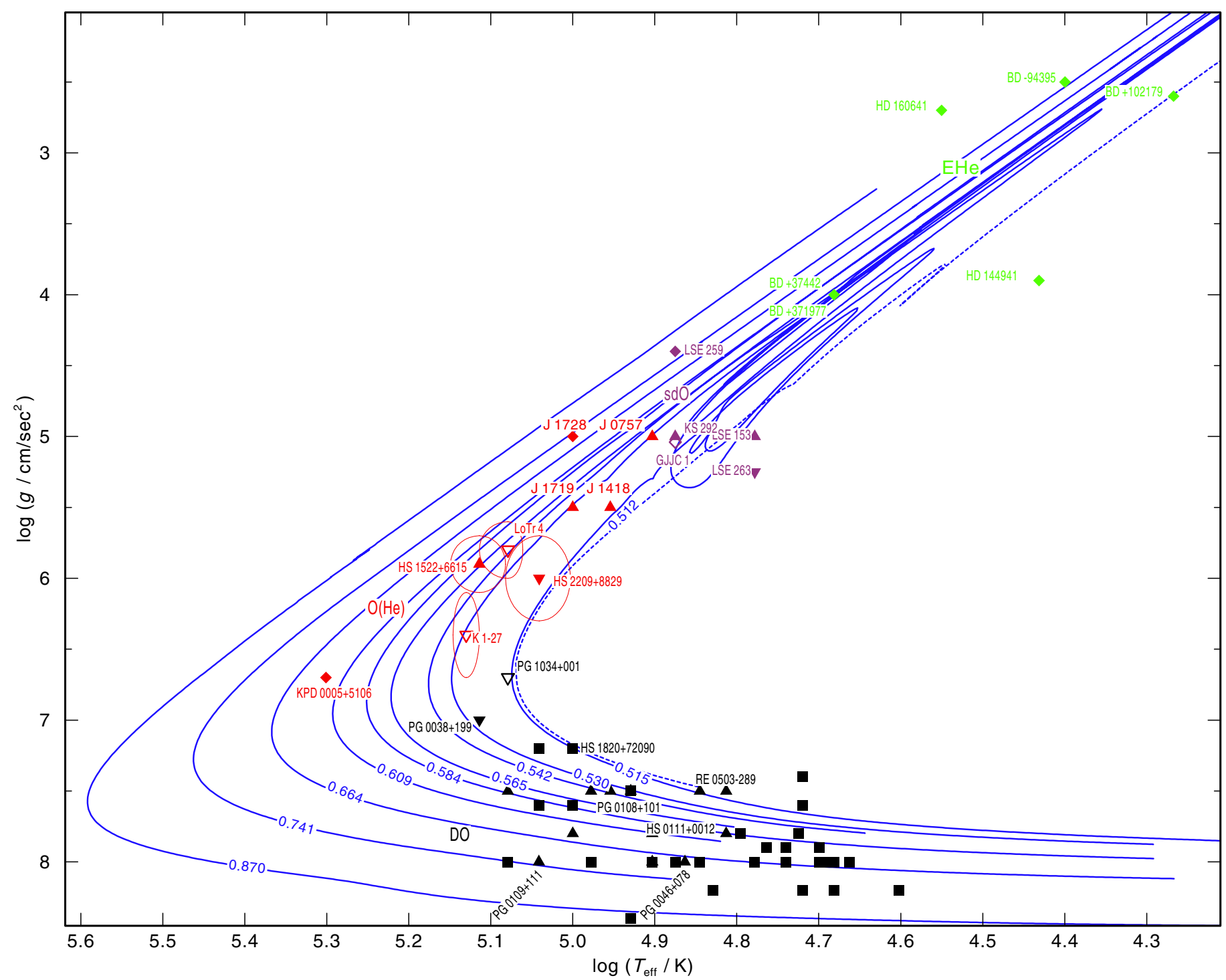

Fig. 24. Locations of EHe stars (green, Jeffery \& Hamann 2010) the luminous sdO-stars (purple, Müller-Ringat 2013; Rauch et al. 1998, 1991; Husfeld et al. 1989) and $\mathrm{O}(\mathrm{He})$ stars (red, this work, Werner et al. 2014; Wassermann et al. 2010) as well as the DO WDs (black, Werner et al. 2014; Mahsereci 2011; Hügelmeyer et al. 2006; Dreizler \& Werner 1996) in the $\log T_{\text {eff }}-\log g$ plane compared with an LTP (dashed line) and VLTP post-AGB (solid lines) evolutionary tracks (labeled with stellar masses in $M_{\odot}$ ) of Miller Bertolami \& Althaus (2006). Open symbols indicate CSPNe, filled ones indicate that no PN was detected. C-rich objects are represented by triangles, N-rich objects by inverted triangles, C-and N-rich objects by diamonds. Squares specify objects that are neither enriched in C nor in N.

Table 4. Predicted mass-loss rates of the $\mathrm{O}(\mathrm{He})$ stars interpolated from Fig. 6a of Pauldrach et al. (1988, P+88) compared with upper limits determined in our analysis (cf. Fig. 25).

\begin{tabular}{crr}
\hline \hline \multirow{2}{*}{ Object } & \multicolumn{2}{c}{$\log \left(\dot{M} / M_{\odot} / \mathrm{yr}\right)$} \\
\cline { 2 - 3 } & \multicolumn{1}{c}{$\mathrm{P}+88$} & \multicolumn{1}{c}{ this work } \\
\hline K 1-27 & -10.0 & $\leq-9.0$ \\
LoTr 4 & -9.4 & $\leq-9.4$ \\
HS 1522+6615 & -8.8 & $\leq-8.8$ \\
HS 2209+8229 & -10.4 & $\leq-10.4$ \\
\hline
\end{tabular}

exmained all strategic wind lines that are located in the FUSE and COS wavelength ranges (Fig. 25) and found that only the mass-loss rate of K 1-27, which shows a weak P-Cygni profile at Ne VII $\lambda 973.3 \AA$ in the FUSE spectrum, could be at most an order of magnitude higher than predicted by radiative-driven wind theory. The highest possible $\dot{M}$ values for all $\mathrm{O}(\mathrm{He})$ stars are given in Table 4.

\section{Stellar parameters and distances}

To derive the stellar parameters, we compared the position of the $\mathrm{O}(\mathrm{He})$ stars in the $\log T_{\text {eff }}-\log g$ plane with different evolutionary tracks. Figure 24 shows the location of the $\mathrm{O}(\mathrm{He})$ stars and related He-dominated objects compared with an LTP evolutionary track (Miller Bertolami \& Althaus 2007) and VLTP post-AGB tracks (Miller Bertolami \& Althaus 2006). The LTP and VLTP tracks only differ at low surface gravities, and the masses derived from H-rich post-AGB evolutionary tracks (Miller Bertolami \& Althaus 2007) also agree well within the error limits with the VLTP masses. Therefore it is justified to derive the masses of the $\mathrm{O}(\mathrm{He})$ stars from these tracks although the VLTP scenario cannot be valid for these stars, as we show below. 


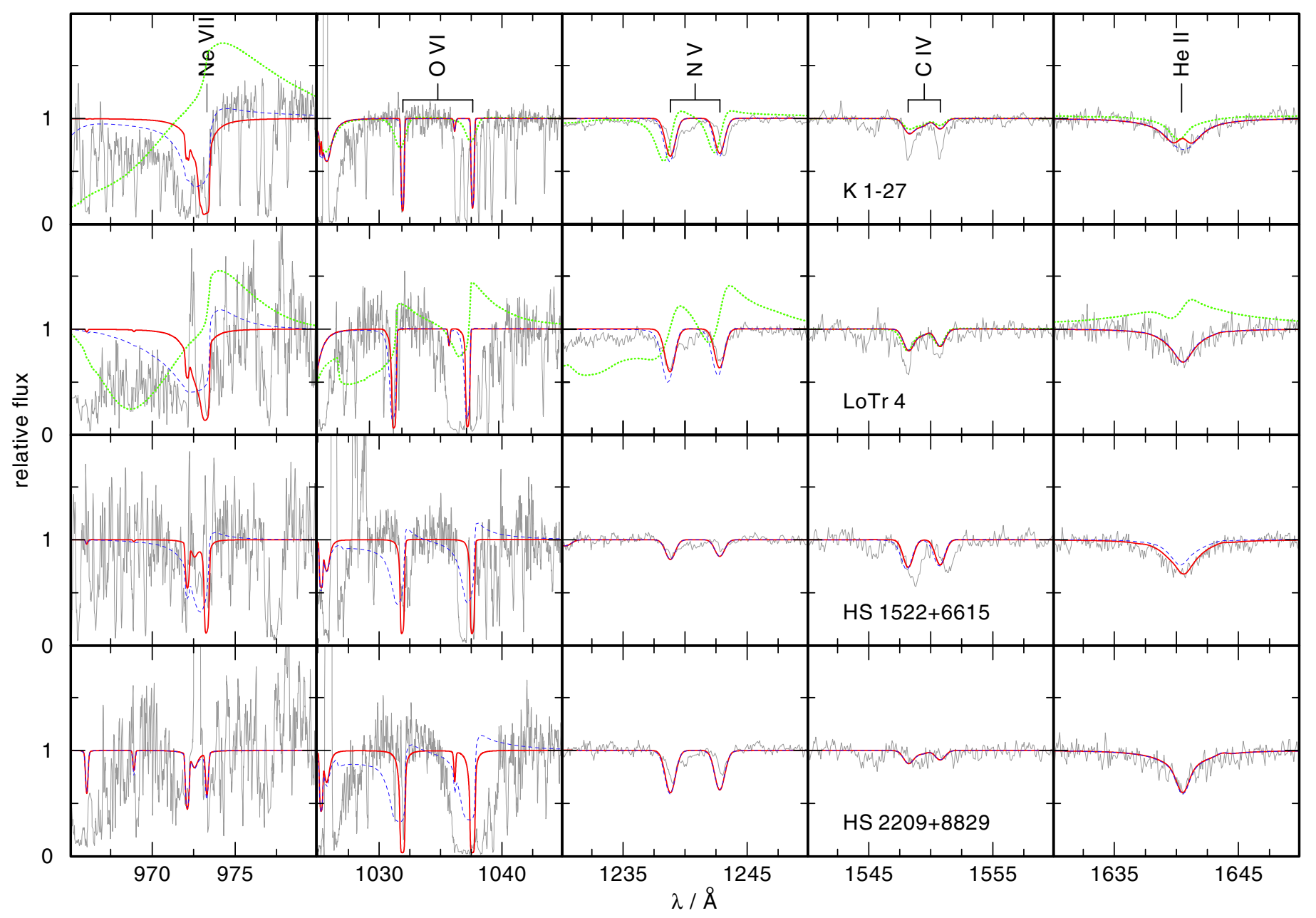

Fig. 25. Synthetic PoWR line profiles of Ne VII $2 p^{1} P^{o}-2 p{ }^{1} \mathrm{D}$, of the resonance doublets of O VI, N v, C IV, and of He II $\lambda 1640 \AA$ calculated with mass-loss rates from Pauldrach et al. (1988, red lines) and $10 \times$ and $100 \times($ K 1-27 and LoTr 4 only) enhanced mass-loss rates (blue, dashed and green, dotted lines, respectively), compared with the observations of the four $\mathrm{O}(\mathrm{He})$ stars.

We also derived the stellar parameters of the $\mathrm{O}(\mathrm{He})$ stars using the evolutionary tracks for double He-WD mergers (Zhang \& Jeffery 2012b, Fig. 26). For K 1-27, LoTr 4, and HS 2209+8229, we used the tracks from the slow-merger models because they reproduce the surface abundances of theses stars. For HS 1522+6615 the fast-merger models were used, because they result in C-enriched atmospheres (Sect. 5.2.1). Compared with what we found by comparison with VLTP calculations, luminosities and radii, which were derived from merger processes agree quite well, but the masses are up to $\Delta M=0.16 M_{\odot}$ higher (Table 5).

Based on these masses, we calculated the distances (Table 5) by using the flux calibration of Heber et al. (1984) for $\lambda_{\mathrm{eff}}=$ $5454 \AA$,

$d[\mathrm{pc}]=7.11 \times 10^{4} \cdot \sqrt{H_{v} \cdot M \times 10^{0.4 m_{v_{0}}-\log g}}$,

with $m_{\mathrm{V}_{\mathrm{o}}}=m_{\mathrm{V}}-2.175 c, c=1.47 E_{B-V}$, and the Eddington flux $H_{v}$ at $\lambda_{\text {eff }}$ of our final model atmospheres. All $\mathrm{O}(\mathrm{He})$ stars and in particular HS $1522+6615$ are located far above or below the Galactic plane (Table 5). K 1-27, LoTr 4, and HS 2209+8229 may belong to the thick disk, which dominates in the region $1 \mathrm{kpc} \lesssim z \lesssim 4 \mathrm{kpc}$ (Kordopatis et al. 2011). HS 1522+6615 has a high radial velocity of $-180 \mathrm{~km} \mathrm{~s}^{-1}$ (measured by the shift of e.g. C IV $\lambda \lambda 1168.9,1169.0 \AA$ and He II $\lambda 1640.4 \AA$ in the COS spectrum). Using the proper motions from the Sloan
Table 5. Masses $M_{\mathrm{m}}$ and $M_{\mathrm{V}}$ interpolated from double He-WD mergers (Fig. 26) and evolutionary tracks of VLTP post-AGB stars (Fig. 24), respectively, luminosities $L$, radii $R$, Galactic coordinates $l$ and $b$, distances $d$, and heights above the Galactic plane $z$ of the $\mathrm{O}(\mathrm{He})$ stars, as well as the kinematical ages $t_{\mathrm{PN}}$ of the two PNe.

\begin{tabular}{rcccc}
\hline \hline & K 1-27 & LoTr 4 & HS & HS \\
& & HS 6615 & $2209+8829$ \\
\hline$M_{\mathrm{m}} / M_{\odot}$ & $0.60_{-0.03}^{+0.08}$ & $0.70_{-0.05}^{+0.08}$ & $0.70_{-0.05}^{+0.05}$ & $0.60_{-0.05}^{+0.10}$ \\
$M_{\mathrm{V}} / M_{\odot}$ & $0.53_{-0.01}^{+0.03}$ & $0.54_{-0.01}^{+0.10}$ & $0.57_{-0.03}^{+0.07}$ & $0.52_{-0.01}^{+0.02}$ \\
$\log L / L_{\odot}$ & $3.2 \pm 0.4$ & $3.6 \pm 0.3$ & $3.7 \pm 0.2$ & $3.2 \pm 0.2$ \\
$R / R_{\odot}$ & $0.08_{-0.03}^{+0.03}$ & $0.14_{-0.03}^{+0.05}$ & $0.14_{-0.02}^{+0.06}$ & $0.12_{-0.04}^{+0.07}$ \\
$l /^{\circ}$ & 286.877 & 291.434 & 102.481 & 117.849 \\
$b /^{\circ}$ & -29.577 & +19.258 & +44.561 & +21.545 \\
$d / \mathrm{kpc}$ & $2.00_{-0.84}^{+0.63}$ & $4.27_{-1.15}^{+1.00}$ & $7.86_{-2.27}^{+1.72}$ & $2.90_{-1.24}^{+0.90}$ \\
$z / \mathrm{kpc}$ & $-0.99_{+0.41}^{-0.31}$ & $1.41_{-0.38}^{+0.33}$ & $5.51_{-1.59}^{+1.21}$ & $1.07_{-0.45}^{+0.33}$ \\
$t_{\mathrm{PN}} / \mathrm{yr}$ & $10867_{-4553}^{+3421}$ & $16184_{-4360}^{+3794}$ & & \\
\hline
\end{tabular}

Digital Sky Survey Photometric Catalog (Release 9, Ahn et al. 2012), we calculated the space velocities to $U=95 \mathrm{~km} \mathrm{~s}^{-1}$, $V=-116 \mathrm{~km} \mathrm{~s}^{-1}$, and $W=-121 \mathrm{~km} \mathrm{~s}^{-1}$, which are typical for halo stars (Kordopatis et al. 2011). From the large height above 


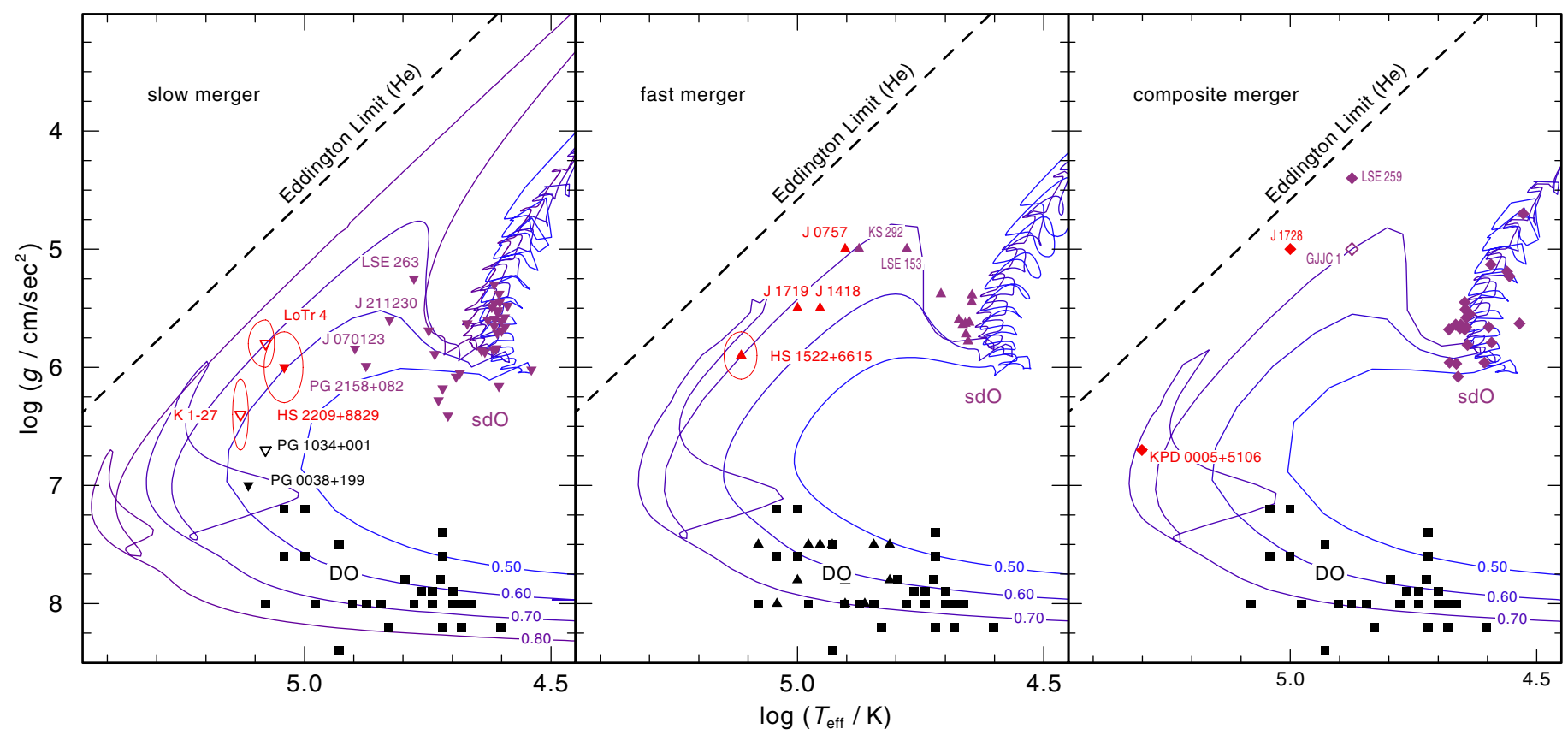

Fig. 26. Evolutionary tracks for a slow-merger (left), a fast-merger (middle) and a composite-merger (right) scenario of two He-WDs, marked with the merger mass in $M_{\odot}$ (Zhang \& Jeffery 2012b). The locations of the sdB-, sdO-stars, (purple, Müller-Ringat 2013; Németh et al. 2012; Naslim et al. 2010; Hirsch 2009; Ahmad et al. 2004; Rauch et al. 1998, 1991; Husfeld et al. 1989; Stroeer et al. 2007) and O(He) stars (red this work, Werner et al. 2014; Wassermann et al. 2010) as well as the DO WDs (black, Werner et al. 2014; Mahsereci 2011; Hügelmeyer et al. 2006; Dreizler \& Werner 1996) are marked. Open symbols indicate CSPNe, filled ones that no PN was detected. C-rich objects are represented by triangles, $\mathrm{N}$-rich objects by inverted triangles, $\mathrm{C}$ - and N-rich objects by diamonds. Squares specify objects that are neither enriched in $\mathrm{C}$ nor in N.

the Galactic plane $(z=5.51 \mathrm{kpc})$ of HS $1522+6615$ and its fast space velocities, we conclude that it belongs to the Galactic halo.

Using a typical expansion velocity of $20 \mathrm{~km} \mathrm{~s}^{-1}$ for the PNe of K 1-27 and LoTr 4, we also calculated their kinematical ages. The results are given in Table 5.

\section{Results and discussion}

We re-analyzed all known $\mathrm{O}(\mathrm{He})$ stars based on the available spectra and improved the determination of their properties (Tables 3, 5). We found that $\mathrm{C}, \mathrm{N}, \mathrm{O}$, and Ne have a strong impact on the atmospheric structure and thus a strong effect on the resulting line profiles. This mostly affected K $1-27$, for which we found a $30 \mathrm{kK}$ higher $T_{\text {eff }}$ than in literature. The COS observations allowed us to determine the $\mathrm{N}$ and $\mathrm{O}$ abundances for HS 2209+8229 for the first time. The FUSE observation of K 1-27 allowed us to determine the $\mathrm{C}$ abundances for this star for the first time. We derived upper abundance limits for $\mathrm{F}, \mathrm{Ne}$, Si, P, S (for LoTr 4 and HS 2209+8229 the actual abundance values for $\mathrm{P}$ and $\mathrm{S}$, measured from the FUSE spectrum), Ar, and Fe.

By examining all strategic wind-lines located in the FUSE and COS wavelength ranges, we found that the upper limits for mass-loss rates of the $\mathrm{O}(\mathrm{He})$ stars agree well with predictions by radiative-driven wind theory. Only K 1-27 might have a ten times higher mass-loss rate than predicted (only there did we find spectral signatures). From comparison with stellar-evolution calculations we found that the masses of the $\mathrm{O}(\mathrm{He})$ stars range from $0.52 M_{\odot}$ to $0.57 M_{\odot}$ considering (V)LTP tracks, or from $0.60 M_{\odot}$ to $0.70 M_{\odot}$ considering double He-WD merger tracks.

Before considering possible evolutionary scenarios, it is worth to mention, that the $\mathrm{CNO}$ abundance patterns are similar for all $\mathrm{O}(\mathrm{He})$ stars exept HS 1522+6615 (Fig. 20), which exhibits strongly deviating CNO abundances. This suggests a dichotomy within the $\mathrm{O}(\mathrm{He})$ class. Interestingly, we find that other
He-dominated objects (e.g. He-rich subdwarfs; Müller-Ringat 2013; Németh et al. 2012; Naslim et al. 2010; Hirsch 2009; Ahmad et al. 2004; Rauch et al. 1998, 1991; Husfeld et al. 1989; Stroeer et al. 2007, DO WDs Werner et al. 2014; Mahsereci 2011; Hügelmeyer et al. 2006; Dreizler \& Werner 1996) also show these differences in the $\mathrm{CNO}$ abundances ${ }^{13}$.

Some objects are enriched in $\mathrm{N}$ but not in $\mathrm{C}$, some C-enriched objects do not show $\mathrm{N}$, but there also are objects which are enriched in both. This suggests that there exist subclasses within the He-dominated objects. Each of them might have a different evolutionary history. From the He-dominated post-AGB objects (Fig. 24), it seems that there is no correlation of the stellar mass and the C-enriched objects. There is no star among $\mathrm{N}$-enriched objects with a higher mass than $0.542 \mathrm{M}_{\odot}$. This might be because for low-mass stars no third drege-up is predicted during the AGB evolution (Mello et al. 2012). The Cand $\mathrm{N}$-enriched $\mathrm{sdO}$ and $\mathrm{O}(\mathrm{He})$ stars have masses greater than $0.64 M_{\odot}$.

Rauch et al. (1998) already proposed the $\mathrm{sdO}(\mathrm{He}) \rightarrow \mathrm{O}(\mathrm{He}) \rightarrow \mathrm{DO}$ WD

evolutionary channel that runs parallel to the PG 1159 evolution in the HRD. However, they were unable to present stringent clues on the evolution of $\mathrm{O}(\mathrm{He})$ stars. In the following we discuss different evolutionary channels to explain the origin of the $\mathrm{O}(\mathrm{He})$ stars, but also investigate the origin of other He-dominated objects to find possible progenitors and successors. First we will mention single-star evolutionary channels (Sect.5.1), such as enhanced mass-loss of post early-AGB stars, (V)LTP scenarios, and the hot-flasher scenario. In Sect. 5.2 we consider to formation channels in binary systems, starting with the merger

\footnotetext{
${ }^{13}$ A list of all analyzed He-rich sdO stars, that are enriched in $\mathrm{N}$ and/or $\mathrm{C}, \mathrm{O}(\mathrm{He})$ stars and DO WDs can be found at http://astro. uni-tuebingen.de/ reindl/He
} 
scenarios (Sect. 5.2.1). We also discuss problems of this formation channel and some other possible binary formation channels such as enhanced mass-loss triggered by a planet or brown dwarf and the merger of a He and a CO WD (Sect. 5.2.2).

\subsection{Single-star evolution}

Assuming single-star evolution, Rauch et al. (2009) suggested that low-mass $\mathrm{O}(\mathrm{He})$ stars might be post early-AGB stars that experienced an enhanced mass-loss which removed the H-rich envelope. In these stars, the first thermal pulse occurs after their departure from the AGB. A numerical experiment for a $0.512 M_{\odot}$ star by Miller Bertolami \& Althaus (2006) has shown that an artificially increased mass-loss rate can cause the hydrogen deficiency and, in the later evolution, might turn the $\mathrm{O}(\mathrm{He})$ stars into PG 1159 stars. However, to achieve that Miller Bertolami \& Althaus (2006) had to assume $\log \dot{M} /\left(M_{\odot} / \mathrm{yr}\right)=-8$ for $\log T_{\text {eff }}>3.8$. Such a strong wind is not predicted by radiativedriven wind theory nor is it seen in the spectra of the $\mathrm{O}(\mathrm{He})$ stars. Only K 1-27 could have a ten times higher mass-loss rate than predicted, but the derived upper limited $\left(\log \dot{M} /\left(M_{\odot} /\right.\right.$ yr $\left.)=-9\right)$ is still too low to overcome the He-buffer and consequently turn the $\mathrm{O}(\mathrm{He})$ star into a PG 1159 star. The previous mass-loss history of the $\mathrm{O}(\mathrm{He})$ stars is of course unknown and we can therefore only rule out PG 1159 stars as possible successors of $\mathrm{O}(\mathrm{He})$ stars. However, the different $\mathrm{H}$-abundances of the $\mathrm{N}$-enriched $\mathrm{O}(\mathrm{He})$ stars, show a correlation with stellar mass and hence the post-AGB times and the remaining $\mathrm{H}$ in the atmospheres of these stars. While LoTr 4 still shows $12 \% \mathrm{H}$ (by mass), the more evolved and lower mass K 1-27 only shows $5 \% \mathrm{H}$. No $\mathrm{H}$ can be detected in the spectrum in the lowest mass $\mathrm{O}(\mathrm{He})$ star, HS 2209+8229. Although the predicted mass-loss rates are lower for stars with lower mass, their evolutionary timescales are much longer, and that is why the correlation of stellar mass and remaining $\mathrm{H}$ seems plausible if enhanced mass-loss is assumed.

Within this scenario, good candidates for successors are C-enriched DO WDs (e.g. PG0108+101, HS 0111+0012, Dreizler \& Werner 1996) for HS $1522+6615$ or N-enriched DO WDs (e.g. PG 0038+199, PG 1034+001, Mahsereci 2011) for the three other $\mathrm{O}(\mathrm{He})$ stars. However, because of gravitational settling they might also turn into DOs, which do not show $\mathrm{C}$ or $\mathrm{N}$ anymore, or even, depending on the remaining $\mathrm{H}$, into DA WDs.

Possible progenitors might be luminous helium-rich sdOtype stars, for example LSE 153 (Müller-Ringat 2013) for HS $1522+6615$ or LSE 263 (Müller-Ringat 2013) for the Nenriched $\mathrm{O}(\mathrm{He})$ stars.

Other interesting objects that fit into this sequence, might be the [WN]-type CSPNe IC 4663 (Miszalski et al. 2012) and Abell 48 (Todt et al. 2013; Frew et al. 2014). Because of the strong similarity of the element abundances of [WN] stars and the $\mathrm{N}$-enriched $\mathrm{O}(\mathrm{He})$ stars, an analog to the $\mathrm{H}$-deficient, but C-rich post-AGB sequence [WCL] $\rightarrow$ [WCE] $\rightarrow$ PG 1159 (e.g. Werner \& Herwig 2006), a second, H-deficient sequence $[\mathrm{WN}] \rightarrow \mathrm{O}(\mathrm{He})$ was suggested (cf. Werner 2012). By examining He II $\lambda 4686 \AA$ and Ne VII $\lambda 3890 \AA$, Miszalski et al. (2012) determined $T_{\text {eff }}=140 \mathrm{kK}$ for IC 4663 . Their values for the mass and the surface gravity $\left(M=0.6 M_{\odot}\right.$ and $\left.\log g=6.1\right)$ are only estimates, however. With $T_{\text {eff }}=140 \mathrm{kK}$, IC 4663 would be in a similar or even later evolutionary state than the $\mathrm{O}(\mathrm{He})$ stars. Abell $48\left(T_{\text {eff }}=70 \mathrm{kK}\right)$, on the other hand, is in an earlier evolutionary stage than the $\mathrm{O}(\mathrm{He})$ stars. Still, it is necessary to clarify why IC 4663 and Abell 48 show a much stronger stellar wind
(IC 4663: $\log \dot{M} / M_{\odot} /$ yr $=-7.7$, Abell 48: $\log \dot{M} / M_{\odot} /$ yr $\left.=-6.4\right)$ than the $\mathrm{O}(\mathrm{He})$ stars. We speculate that $[\mathrm{WN}]$ stars are $\mathrm{O}(\mathrm{He})$ stars with higher masses. According to Pauldrach et al. (1988, Fig. 6b), the high mass-loss rate found for IC 4663 and Abell 48 would correspond to $M \approx 0.7 M_{\odot}$ and $M>1.0 M_{\odot}$, respectively.

Rauch et al. (2008) suggested that RCB stars might be possible progenitors of the $\mathrm{O}(\mathrm{He})$ stars. This could be true for the two $\mathrm{C}$ and $\mathrm{N}$ enriched $\mathrm{O}(\mathrm{He})$ stars SDSS J 172854.34+361958.62 (Werner et al. 2014) and KPD 0005+5106 (Wassermann et al. 2010). In between evolutionary objects could be the C- and N-enriched luminous sdO stars LSE 259 (Husfeld et al. 1989), GJJC 1 (Rauch et al. 1998), and KS 292 (Rauch et al. 1991).

The VLTP scenarios that were successfully applied to explain the origin of the H-deficient, but C-rich objects (e.g. PG 1159 stars) cannot explain the origin of the $\mathrm{O}(\mathrm{He})$ stars because these scenarios always produce $\mathrm{C}$-rich atmospheres with more than about $20 \%$ of $\mathrm{C}$ by mass (in contrast, the most $\mathrm{C}$-rich $\mathrm{O}(\mathrm{He})$ stars only show $3 \%$ of $\mathrm{C}$ in their atmospheres). The relatively young kinematical age of the $\mathrm{H}$-rich PNe of K 1-27 and LoTr 4 (around 10000 yrs) strongly contradict a VLTP scenario.

A third conceivable way for a single-star evolutionary channel is the hot-flasher scenario, which was invoked by Miller Bertolami et al. (2008) to explain the helium-rich sdO stars. This scenario is able to explain C- or N-enriched HesdOs, but these stellar models never reach the high effective temperatures found for the $\mathrm{O}(\mathrm{He})$ stars.

\subsection{Binary evolution}

Since none of the previously mentioned single-star formation channels seems convincing for the $\mathrm{O}(\mathrm{He})$ stars, binary formation channels become interesting. We discuss merger scenarios and suggest alternatives.

\subsubsection{Merger scenarios}

As mentioned above, Zhang \& Jeffery (2012b,a) showed that in terms of $T_{\text {eff }}, \log g, \mathrm{C}$, and $\mathrm{N}$ abundances, the origin of the three sdOs groups, but also the properties of RCB and EHe stars, can be explained by different double He-WD merger models. Zhang \& Jeffery (2012a) compared their result with He-rich sdO stars from the sample of Hirsch (2009) and He-rich sdB stars from the sample Naslim et al. (2010). We extended this comparison to all He-rich $\mathrm{sdB}$ and sdO stars, that are enriched in C and/or N (Müller-Ringat 2013; Németh et al. 2012; Naslim et al. 2010; Hirsch 2009; Ahmad et al. 2004; Rauch et al. 1998, 1991; Husfeld et al. 1989; Stroeer et al. 2007), the $\mathrm{O}(\mathrm{He})$ stars of our work, and those of Werner et al. (2014); Wassermann et al. (2010), and the known DO WDs (Werner et al. 2014; Mahsereci 2011; Hügelmeyer et al. 2006; Dreizler $\&$ Werner 1996). We found that all of them fit this scenario. Figure 27 shows the $\mathrm{CNO}$ abundances resulting from a slow and a fast merger of two He WDs according to the numerical experiments of Zhang \& Jeffery (2012a). These abundances are compared with those of the $\mathrm{O}(\mathrm{He})$ stars analyzed in our work. We found that those of the two CSPNe and HS 2209+8229 can be explained by the slow-merger model and the CNO abundances of HS $1522+6615$ are reproduced by the fast-merger because of the high $\mathrm{C}$ abundance. The three $\mathrm{O}(\mathrm{He})$ stars SDSS J 171916.97+365326.70, SDSS J 141812.50+024426.92, and SDSSJ075732.18+184329.28 that were found by Werner et al. (2014) also fit the fast-merger scenario. SDSS J 172854.34+361958.62 (Werner et al. 2014) and 
N. Reindl et al.: On helium-dominated stellar evolution: the mysterious role of the $\mathrm{O}(\mathrm{He})$-type stars

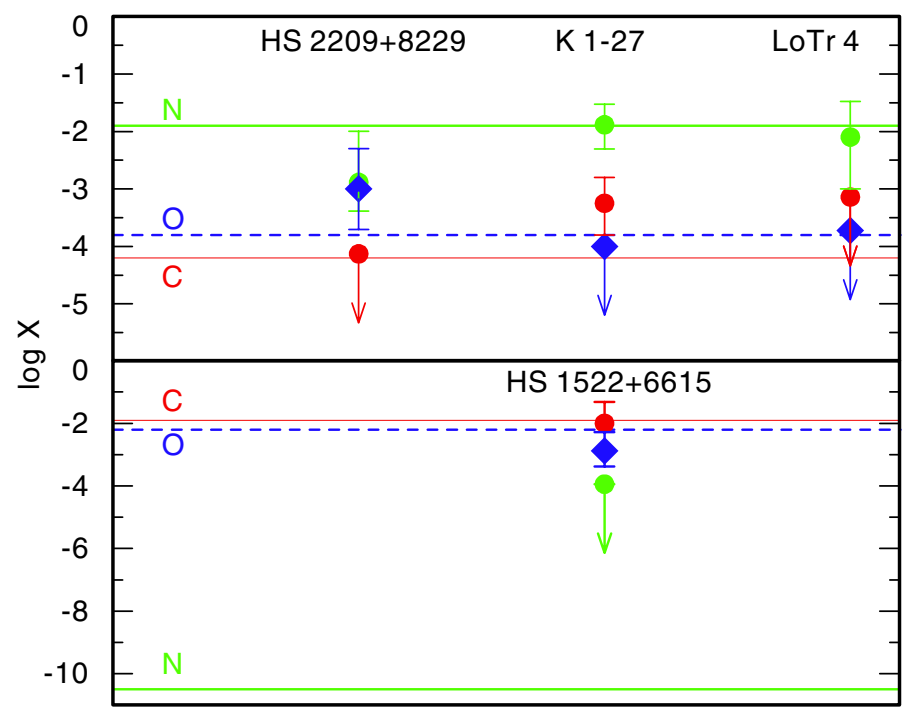

Fig. 27. Comparison of the $\mathrm{CNO}$ abundances of the $\mathrm{O}(\mathrm{He})$ stars $(\mathrm{N}$ : light, green dots, C: dark, red dots, O: dark, blue rhombi) with those resulting form a slow (top) and a fast $\left(0.7 M_{\odot}\right.$ model, bottom $)$ merger of two He WDs (N: light, green, solid line, C: dark, red, solid line, O: dashed, blue, gray line).

KPD 0005+5106 (Wassermann et al. 2010) would fit the composite merger model. Concerning the $\mathrm{CNO}$ abundances, the $\mathrm{O}(\mathrm{He})$ stars can be explained much better by this double $\mathrm{He}$ WD merger scenario than by V(LTP) scenarios.

Figure 26 shows the evolutionary tracks of Zhang \& Jeffery (2012a) for their different merger models. According to their surface abundances, which are produced in a certain merger model, we marked the locations of the $\mathrm{N}$-enriched He-dominated objects in the panel for the slow merger, C-enriched ones in the fast-merger panel, and $\mathrm{C}$ - and $\mathrm{N}$-enriched objects are shown in the composite merger panel. These evolutionary tracks connect the subluminous sdO with the luminous sdO stars (in contrast to the VLTP calculations), the $\mathrm{O}(\mathrm{He})$ stars, and DO WDs.

Other points that support the merger scenario are the very low binary fraction among He-rich sdO-stars (4\%, Napiwotzki et al. 2004), O(He) stars, and DO WDs (only PG 0046+078 and PG $0237+116$ have been found to be in a binary system). A different evolution scenario probably applies for binary systems.

The position of HS $1522+6615$ in the Galactic halo corroborates the merger scenario, because only very old stars are expected there. Napiwotzki (2008) found that a considerable part $(20 \%)$ of the He-rich sdO stars belongs to the halo population. The fact that no PN is detected around HS $1522+6615$, SDSS J 171916.97+365326.70, SDSS J 141812.50+024426.92, and SDSS J 075732.18+184329.28 although their theoretical post-AGB evolutionary times would be shorter than those of K 1-27 or LoTr 4, might be explained considering that these objects are merger products.

We note that the WD+post-sdB star merger channel, proposed by Justham et al. (2011) to explain He-rich sdO stars, can explain the $\mathrm{O}(\mathrm{He})$ stars in terms of $T_{\text {eff }}$ and $\log g$ as well.

\subsubsection{Alternative scenarios}

Within the merger models mentioned above it is not possible to explain the PNe of K 1-27 and LoTr 4. Even if a PN would have been ejected during the merger process, it would have dissipated into the ISM a long time ago because the post-merger time of
LoTr 4 is about $3.7 \times 10^{7}$ years. In addition, the solar compositions of the PNe of $\mathrm{K} 1-27$ and LoTr 4 and the remaining hydrogen in their atmospheres contradicts a merger origin of these objects. The same holds for GJJC 1, IC 4663, and PG 1034+001. For these reasons common envelope (CE) scenarios become interesting. Soker (2013) speculated that R CrB itself and similar $\mathrm{RCB}$ stars are formed from the merger of a WD with the core of an AGB star. The previous common-envelope ejection (CEE) then would have caused the H-deficiency. This scenario would also be possible for the $\mathrm{O}(\mathrm{He})$ stars. The merger within a $\mathrm{CE}$ of a CO-WD and a RG or AGB star might produce a similar outcome as a He- and CO-WD merger but within a $\mathrm{H}$-rich envelope. The $\mathrm{CE}$ ejected during the merger process could then first reproduce the H-rich PNe of K 1-27 and LoTr 4 and then, the post-merger times would be much shorter because no central helium burning phase is expected for a He- and CO-WD merger.

Another possibility for a $\mathrm{O}(\mathrm{He})$ star origin is that an $\mathrm{RG}$ or AGB star lost its H-rich envelope with the help of a planet or a brown dwarf. If a low-mass companion enters the atmosphere of an RG or AGB star, it spirals inwards and transfers orbital energy and angular momentum to the envelope and, thus, parts or all of it are removed. The companion then either stops in a close orbit or even merges with the more massive star (Diehl et al. 2008; Soker 1998).

\subsection{Conclusions}

The evolutionary status of the $\mathrm{O}(\mathrm{He})$ stars, but also those of other He-dominated stars, is still not understood. It is most likely that they are part of a second $\mathrm{H}$-deficient evolutionary sequence. It appears plausible that there are three subclasses within this Hedominated sequence. For objects that are either enriched in $\mathrm{C}$ or $\mathrm{N}$, we propose the channels

$$
\mathrm{sdO}(\mathrm{He}) \rightarrow \mathrm{O}(\mathrm{He}) \rightarrow \mathrm{DO} \text { WD }
$$

or

$$
\mathrm{sdO}(\mathrm{He}) \rightarrow \mathrm{O}(\mathrm{He}) \rightarrow \mathrm{DA} \text { WD }
$$

if there is some remaining $\mathrm{H}$. For $\mathrm{C}$ - and $\mathrm{N}$-rich objects, we propose

$$
\begin{aligned}
\text { or } & \mathrm{RCB} \\
& \rightarrow \mathrm{EHe} \rightarrow \mathrm{sdO}(\mathrm{He}) \rightarrow \mathrm{O}(\mathrm{He}) \rightarrow \mathrm{DO} \text { WD } \\
\mathrm{RCB} & \rightarrow \mathrm{EHe} \rightarrow \mathrm{sdO}(\mathrm{He}) \rightarrow \mathrm{O}(\mathrm{He}) \rightarrow \mathrm{DA} \text { WD }
\end{aligned}
$$

if that there is some remaining $\mathrm{H}$.

This He-dominated sequence has most likely various formation channels. For single-star objects, merger scenarios seem most promising. He-dominated CSPNe must have formed in a different way, for example via enhanced mass-loss during their post-AGB evolution or a merger within a CE of a CO-WD and an RG or AGB star.

To make progress, it would be highly desirable that more $\mathrm{O}(\mathrm{He})$ stars and other He-dominated objects were discovered to improve the statistics. Additional quantitative investigations on the binarity of these objects and on the mass-loss rates of RCB, $\mathrm{EHe}$, and sdO stars may help to distinguish between different formation channels. A comprehensive systematic calculation of evolutionary models for thermal pulse and merger scenarios is a pre-requisite for comparision with results of spectral analysis.

Acknowledgements. N.R. is supported by the German Research Foundation (DFG, grant WE 1312/41-1), T.R. by the German Aerospace Center (DLR, grant 05 OR 1301). We thank Marcelo Miguel Miller Bertolami, Simon Jeffery, Stephan Geier, and Geoffrey Clayton for helpful discussions and comments. This research has made use of the SIMBAD database, operated at CDS, Strasbourg, France. This research has made use of NASA's Astrophysics Data System. This work used the profile-fitting procedure OWENS developed by M. Lemoine and 
the FUSE French Team. Some of the data presented in this paper were obtained from the Mikulski Archive for Space Telescopes (MAST). STScI is operated by the Association of Universities for Research in Astronomy, Inc., under NASA contract NAS5-26555. Support for MAST for non-HST data is provided by the NASA Office of Space Science via grant NNX09AF08G and by other grants and contracts.

\section{References}

Ahmad, A., Jeffery, C. S., \& Fullerton, A. W. 2004, A\&A, 418, 275

Ahn, C. P., Alexandroff, R., Allende Prieto, C., et al. 2012, ApJS, 203, 21 Asplund, M., Grevesse, N., Sauval, A. J., \& Scott, P. 2009, ARA\&A, 47, 481 Bucciarelli, B., García Yus, J., Casalegno, R., et al. 2001, A\&A, 368, 335 Clayton, G. C., Sugerman, B. E. K., Stanford, S. A., et al. 2011, ApJ, 743, 44 Diehl, S., Fryer, C., \& Herwig, F. 2008, in Hydrogen-Deficient Stars, eds. K. Werner \& T. Rauch, ASP Conf. Ser., 391, 221

Dreizler, S., \& Werner, K. 1994, in Evolution of Massive Stars, eds.

D. Vanbeveren, W. van Rensbergen, \& C. De Loore, 147

Dreizler, S., \& Werner, K. 1996, A\&A, 314, 217

Fitzpatrick, E. L. 1999, PASP, 111, 63

Frew, D. J., Bojičić, I. S., Parker, Q. A., et al. 2014, MNRAS, 440, 1345

Gräfener, G., Koesterke, L., \& Hamann, W.-R. 2002, A\&A, 387, 244

Hamann, W.-R., \& Gräfener, G. 2004, A\&A, 427, 697

Han, Z., Podsiadlowski, P., Maxted, P. F. L., Marsh, T. R., \& Ivanova, N. 2002, MNRAS, 336, 449

Han, Z., Podsiadlowski, P., Maxted, P. F. L., \& Marsh, T. R. 2003, MNRAS, 341 , 669

Heber, U., Hunger, K., Jonas, G., \& Kudritzki, R. P. 1984, A\&A, 130, 119 Hébrard, G., \& Moos, H. W. 2003, ApJ, 599, 297

Hébrard, G., Lemoine, M., Vidal-Madjar, A., et al. 2002, ApJS, 140, 103

Hirsch, H. A. 2009, Ph.D. Thesis, University Nuremberg

Hügelmeyer, S. D., Dreizler, S., Homeier, D., et al. 2006, A\&A, 454, 617

Husfeld, D., Butler, K., Heber, U., \& Drilling, J. S. 1989, A\&A, 222, 150

Jeffery, C. S., \& Hamann, W.-R. 2010, MNRAS, 404, 1698

Jeffery, C. S., Karakas, A. I., \& Saio, H. 2011, MNRAS, 414, 3599

Justham, S., Podsiadlowski, P., \& Han, Z. 2011, MNRAS, 410, 984

Kameswara Rao, N., Lambert, D. L., García-Hernández, D. A., \& Manchado, A. 2013, MNRAS, 431, 159

Kordopatis, G., Recio-Blanco, A., de Laverny, P., et al. 2011, A\&A, 535, A107 Lamers, H. J. G. L. M., Snow, T. P., \& Lindholm, D. M. 1995, ApJ, 455, 269

Mahsereci, M. 2011, Diploma Thesis, University Tübingen

Mello, D. R. C., Daflon, S., Pereira, C. B., \& Hubeny, I. 2012, A\&A, 543, A11

Méndez, R. H., Miguel, C. H., Heber, U., \& Kudritzki, R. P. 1986, in Hydrogen

Deficient Stars and Related Objects, eds. K. Hunger, D. Schönberner, \&

N. Kameswara Rao, Astrophys. IAU Colloq. 87, Space Sci. Lib., 128, 323
Mickaelian, A. M., Mikayelyan, G. A., \& Sinamyan, P. K. 2011, MNRAS, 415, 1061

Miller Bertolami, M. M., \& Althaus, L. G. 2006, A\&A, 454, 845

Miller Bertolami, M. M., \& Althaus, L. G. 2007, A\&A, 470, 675

Miller Bertolami, M. M., Althaus, L. G., Unglaub, K., \& Weiss, A. 2008, A\&A, 491, 253

Miszalski, B., Crowther, P. A., De Marco, O., et al. 2012, MNRAS, 423, 934

Müller-Ringat, E. 2013, Dissertation, University of Tübingen, Germany, http://tobias-lib.uni-tuebingen.de/volltexte/2013/6774/

Napiwotzki, R. 2008, in Hydrogen-Deficient Stars, eds. K. Werner \& T. Rauch, ASP Conf. Ser., 391, 257

Napiwotzki, R., Karl, C. A., Lisker, T., et al. 2004, Ap\&SS, 291, 321

Naslim, N., Jeffery, C. S., Ahmad, A., Behara, N. T., \& Şahìn, T. 2010, MNRAS, 409, 582

Németh, P., Kawka, A., \& Vennes, S. 2012, MNRAS, 427, 2180

Pauldrach, A., Puls, J., Kudritzki, R. P., Méndez, R. H., \& Heap, S. R. 1988, A\&A, 207, 123

Rauch, T., \& Deetjen, J. L. 2003, in Stellar Atmosphere Modeling, eds. I. Hubeny, D. Mihalas, \& K. Werner, ASP Conf. Ser., 288, 103

Rauch, T., Heber, U., Hunger, K., Werner, K., \& Neckel, T. 1991, A\&A, 241, 457

Rauch, T., Köppen, J., \& Werner, K. 1994, A\&A, 286, 543

Rauch, T., Köppen, J., \& Werner, K. 1996, A\&A, 310, 613

Rauch, T., Dreizler, S., \& Wolff, B. 1998, A\&A, 338, 651

Rauch, T., Reiff, E., Werner, K., et al. 2006, in Astrophysics in the Far Ultraviolet: Five Years of Discovery with FUSE, eds. G. Sonneborn, H. W. Moos, \& B.-G. Andersson, ASP Conf. Ser., 348, 194

Rauch, T., Reiff, E., Werner, K., \& Kruk, J. W. 2008, in Hydrogen-Deficient Stars, eds. K. Werner \& T. Rauch, ASP Conf. Ser., 391, 135

Rauch, T., Werner, K., \& Kruk, J. W. 2009, in AIP Conf. Ser. 1135, eds. M. E. van Steenberg, G. Sonneborn, H. W. Moos, \& W. P. Blair, 168

Savitzky, A., \& Golay, M. J. E. 1964, Anal. Chem., 36, 1627

Soker, N. 1998, AJ, 116, 1308

Soker, N. 2013, New Astron., 18, 18

Stroeer, A., Heber, U., Lisker, T., et al. 2007, A\&A, 462, 269

Todt, H., Kniazev, A. Y., Gvaramadze, V. V., et al. 2013, MNRAS, 430, 2302

Wassermann, D., Werner, K., Rauch, T., \& Kruk, J. W. 2010, A\&A, 524, A9

Werner, K. 2012, in Planetary Nebulae: An Eye to the Future, IAU Symp., 283, 196

Werner, K., \& Herwig, F. 2006, PASP, 118, 183

Werner, K., Deetjen, J. L., Dreizler, S., et al. 2003, in Stellar Atmosphere Modeling, eds. I. Hubeny, D. Mihalas, \& K. Werner, ASP Conf. Ser., 288, 31

Werner, K., Rauch, T., \& Kepler, S. O. 2014, A\&A, 564, A53

Zhang, X., \& Jeffery, C. S. 2012a, MNRAS, 426, L81

Zhang, X., \& Jeffery, C. S. 2012b, MNRAS, 419, 452 
N. Reindl et al.: On helium-dominated stellar evolution: the mysterious role of the $\mathrm{O}(\mathrm{He})$-type stars

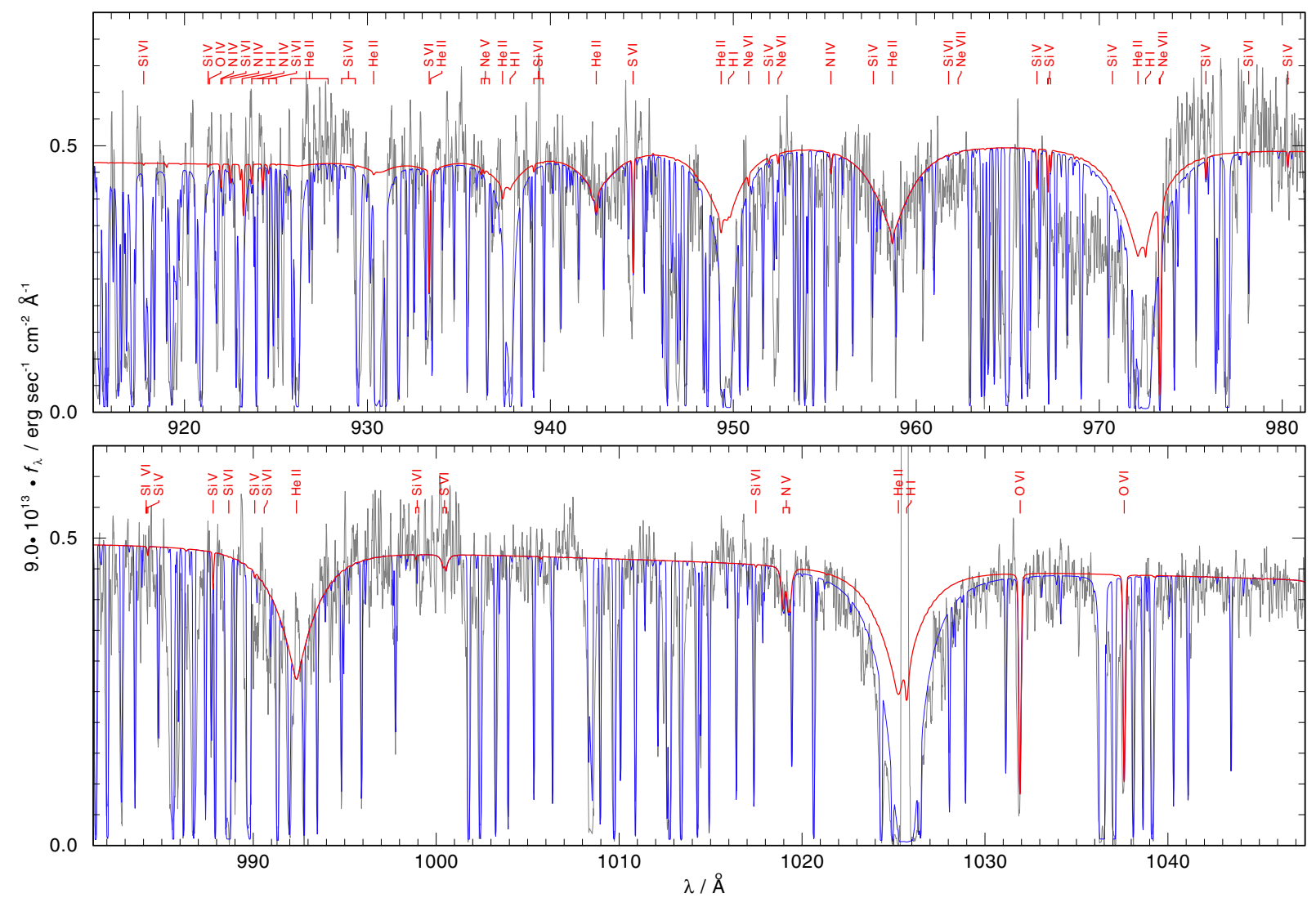

Fig. 1. FUSE spectrum of K 1-27 (gray) compared with final synthetic spectra (red: pure stellar, blue: combined stellar and interstellar). The locations of photospheric lines appearing in the synthetic spectrum are marked.

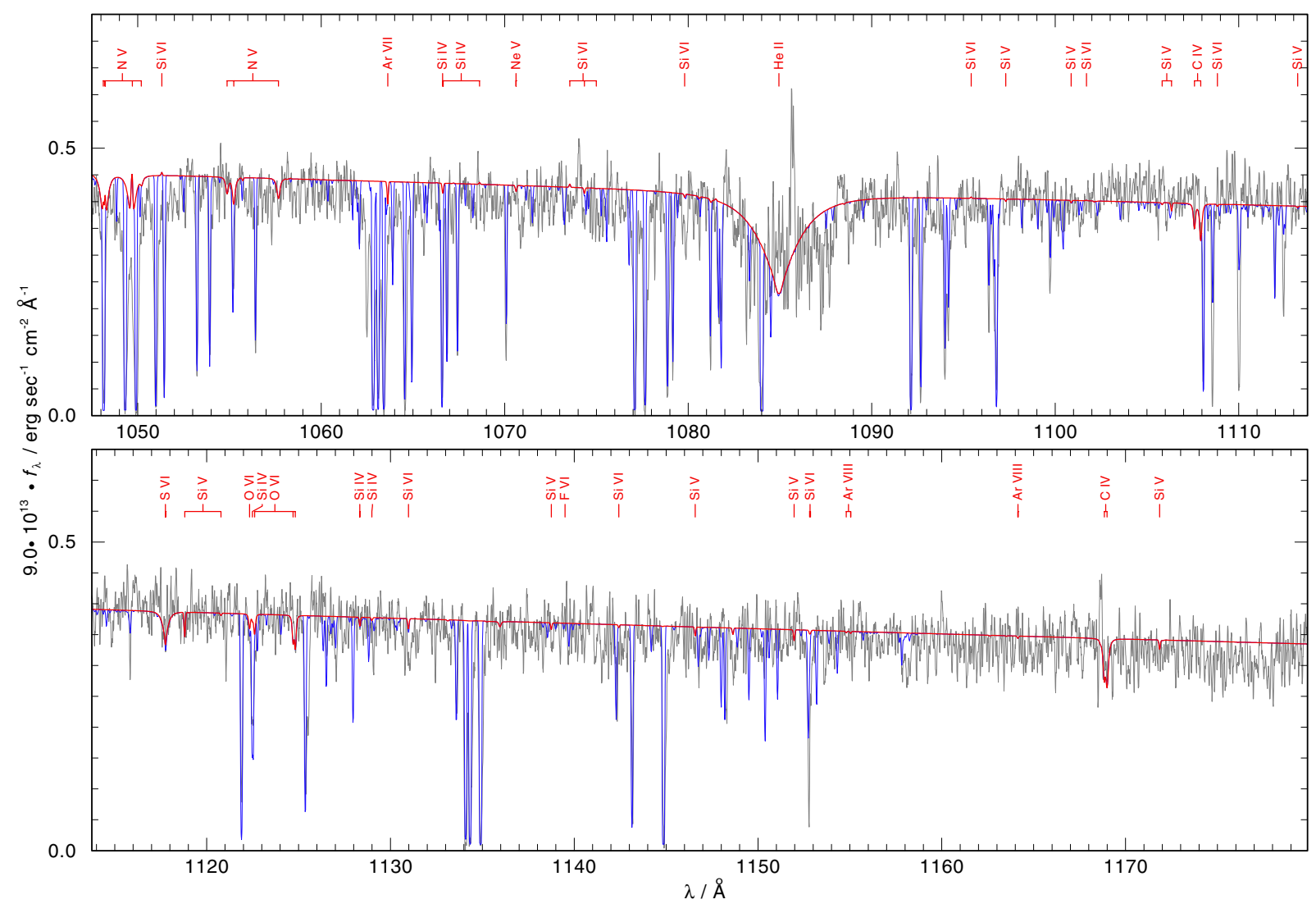

Fig. 1. continued. 
A\&A 566, A116 (2014)

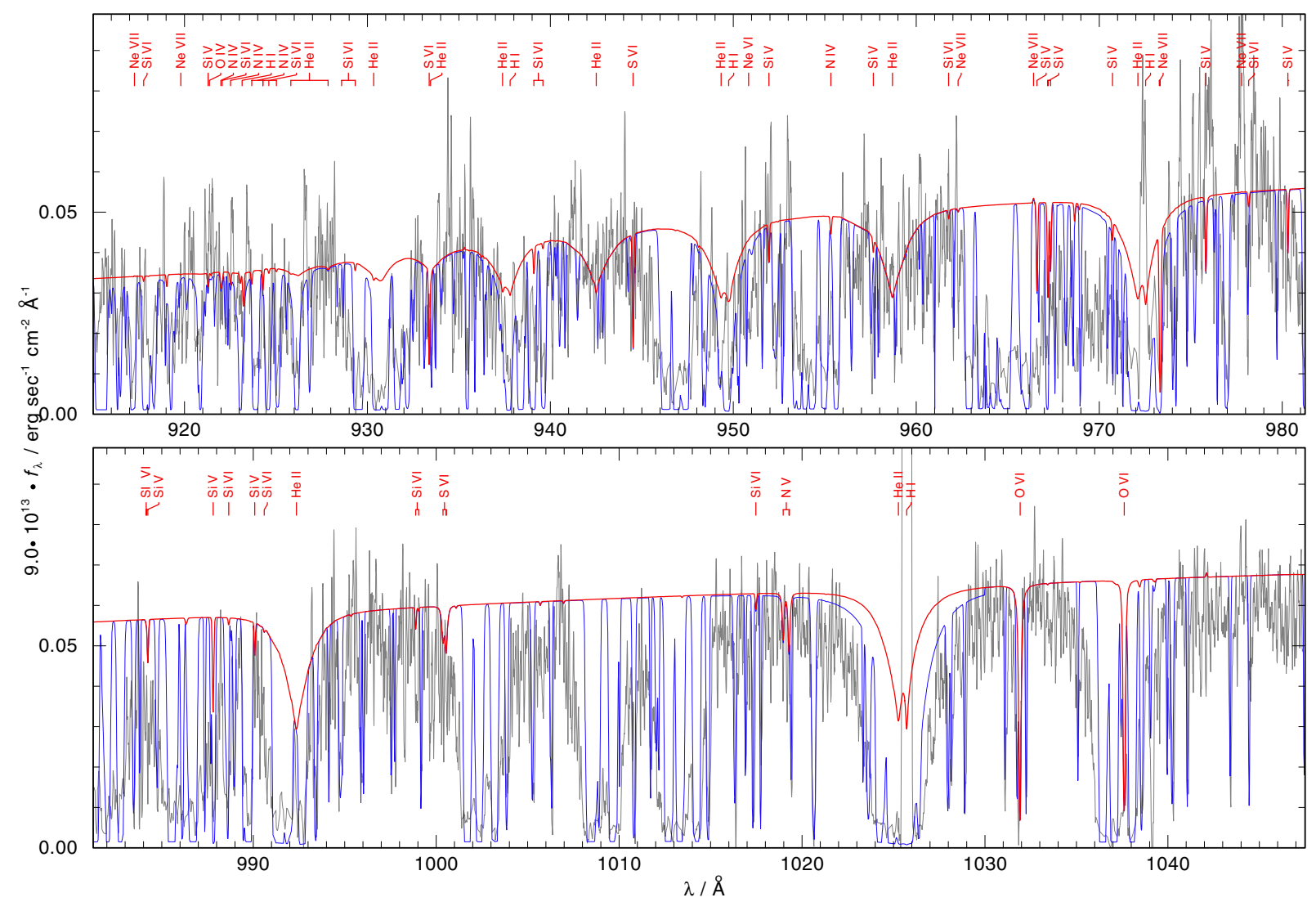

Fig. 2. Same as Fig. 1 for LoTr 4.

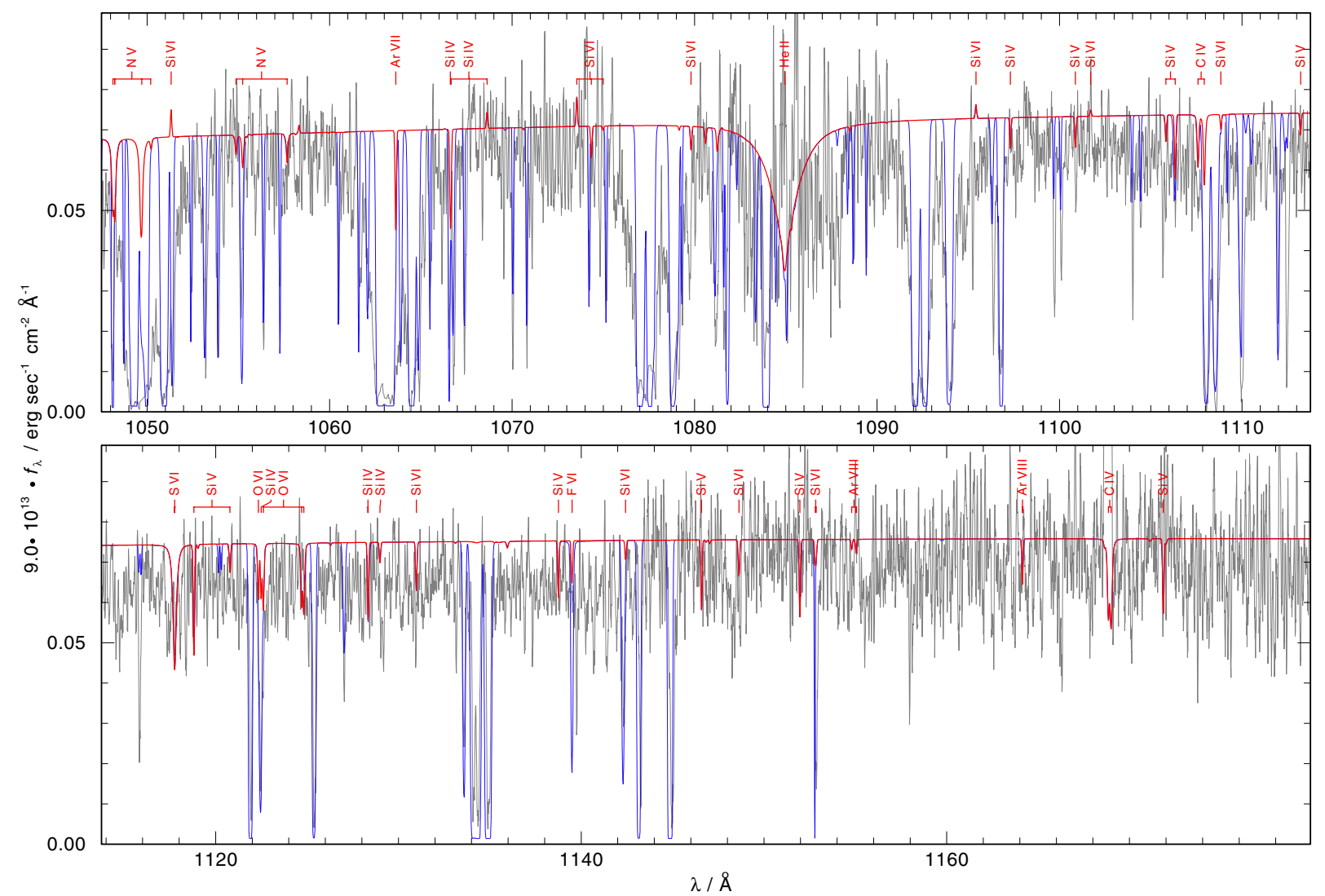

Fig. 2. continued. 
N. Reindl et al.: On helium-dominated stellar evolution: the mysterious role of the $\mathrm{O}(\mathrm{He})$-type stars

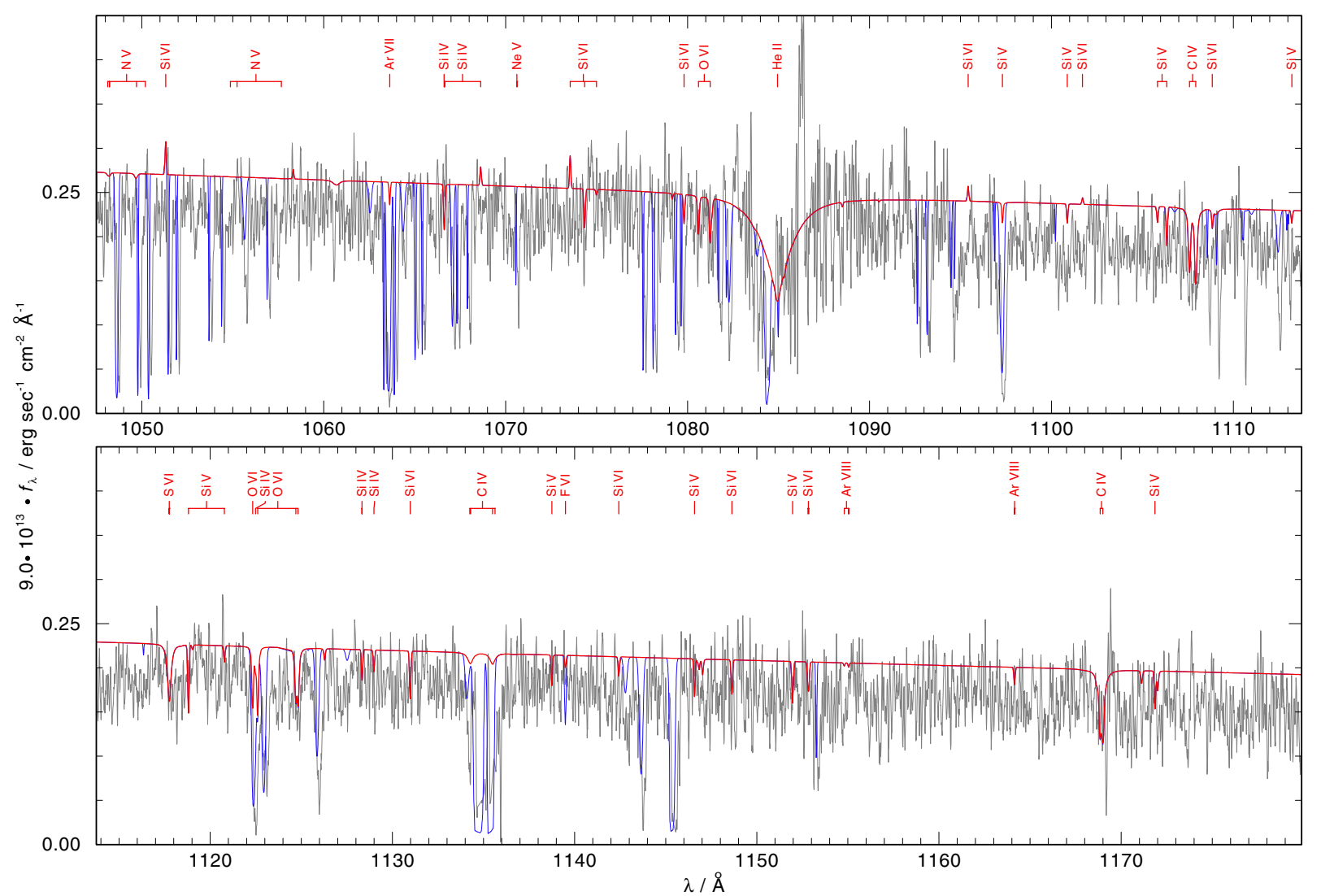

Fig. 3. Same as Fig. 1 for HS $1522+6615$.

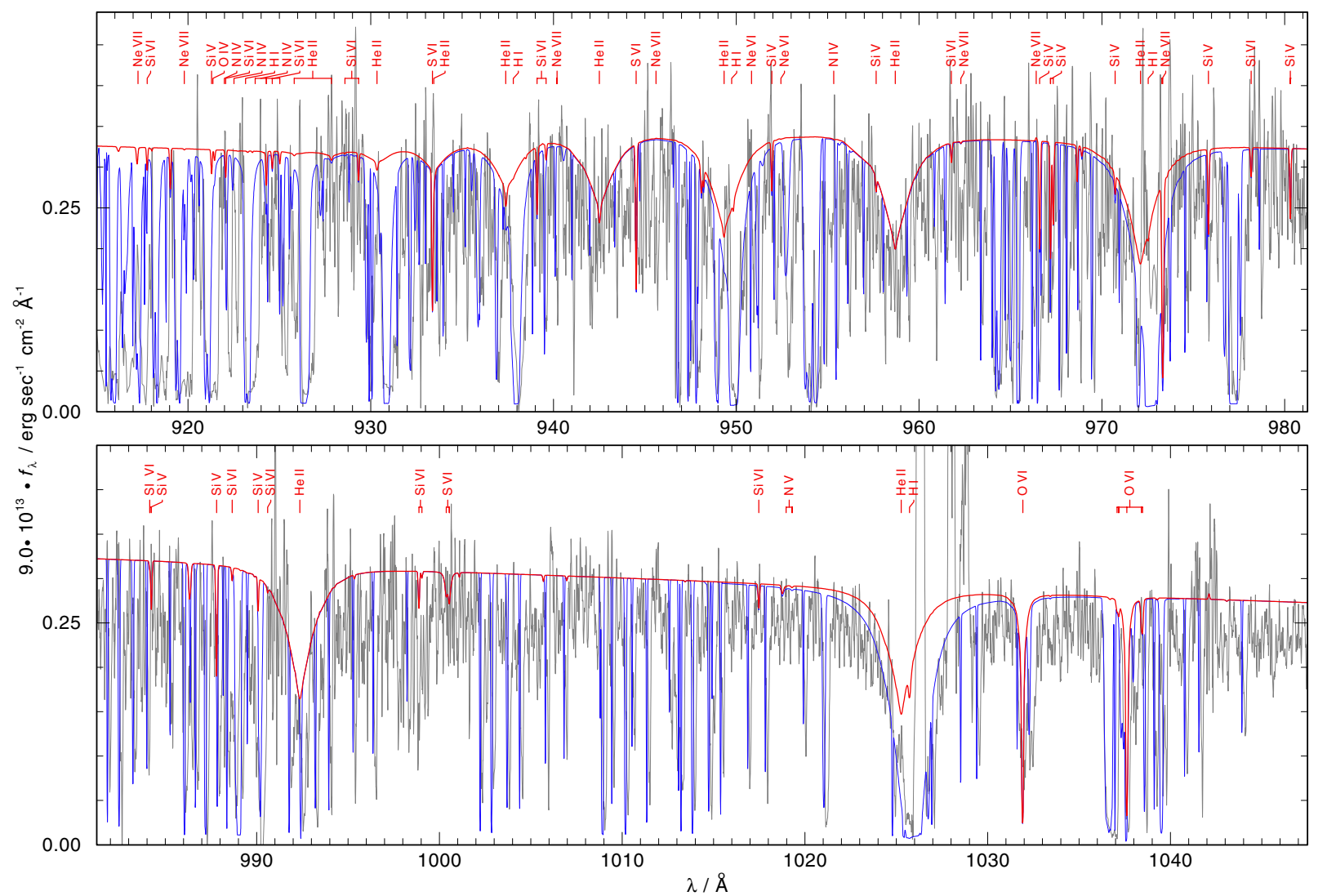

Fig. 3. continued. 
A\&A 566, A116 (2014)

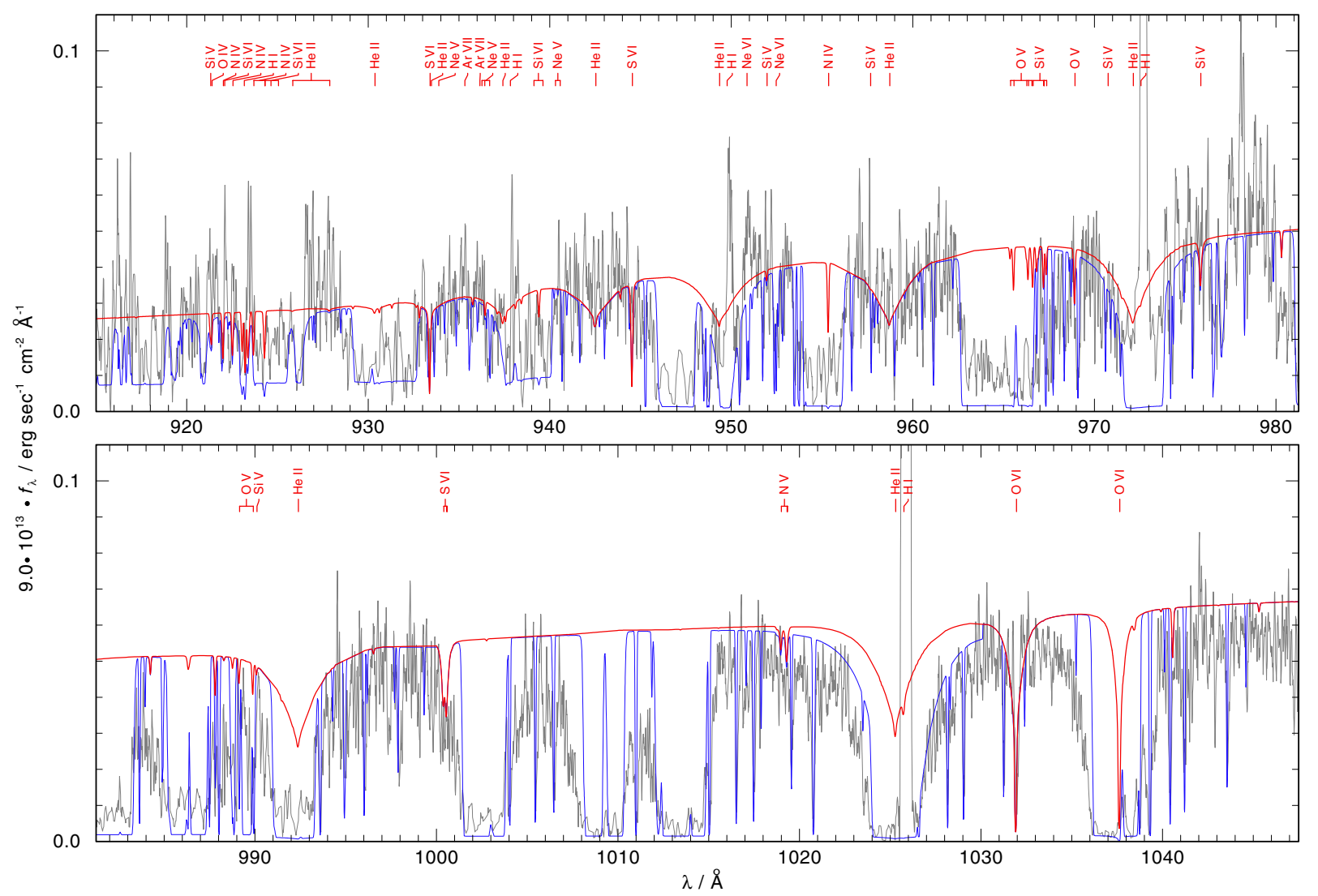

Fig. 4. Same as Fig. 1 for HS 2209+8229.

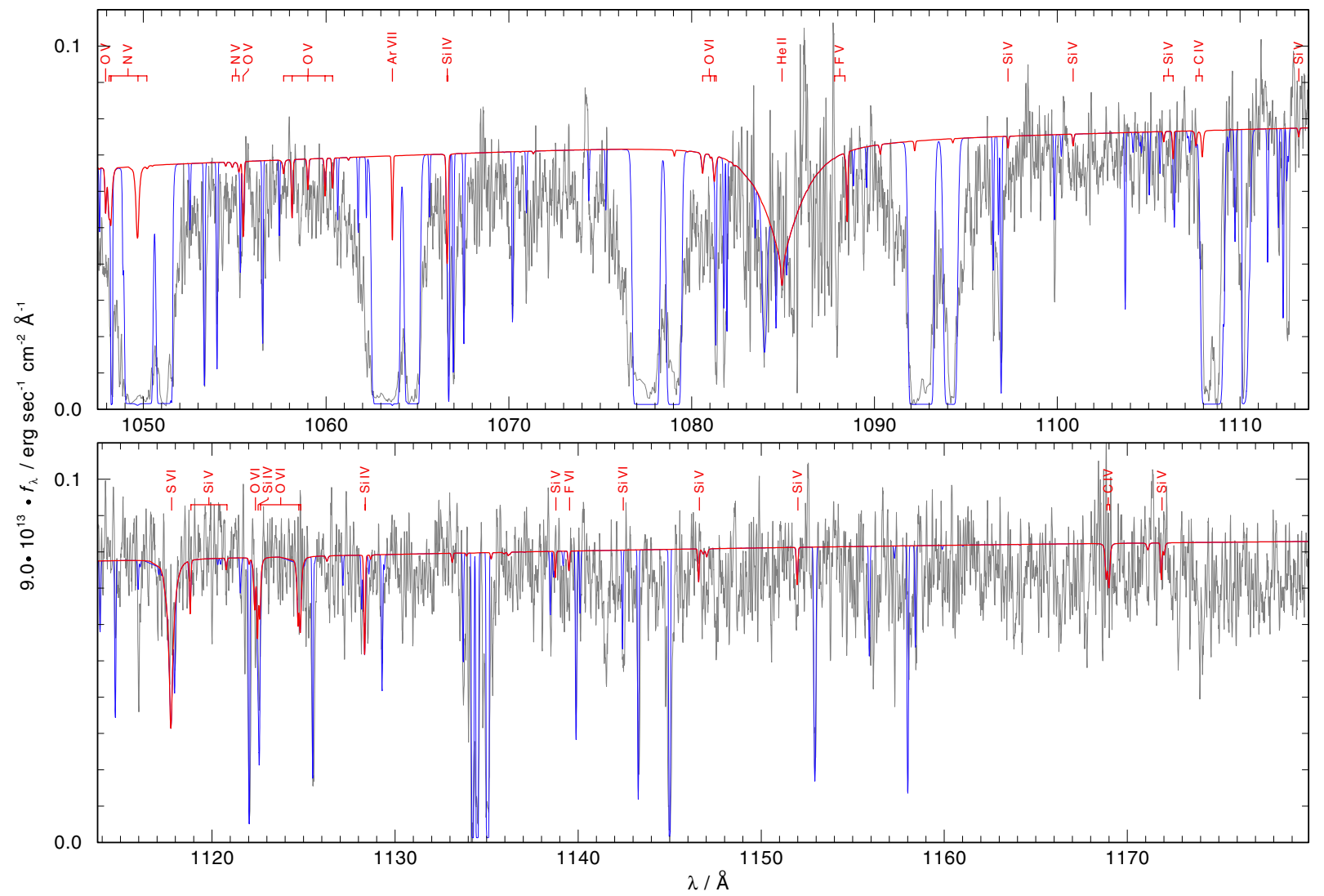

Fig. 4. continued. 
N. Reindl et al.: On helium-dominated stellar evolution: the mysterious role of the $\mathrm{O}(\mathrm{He})$-type stars

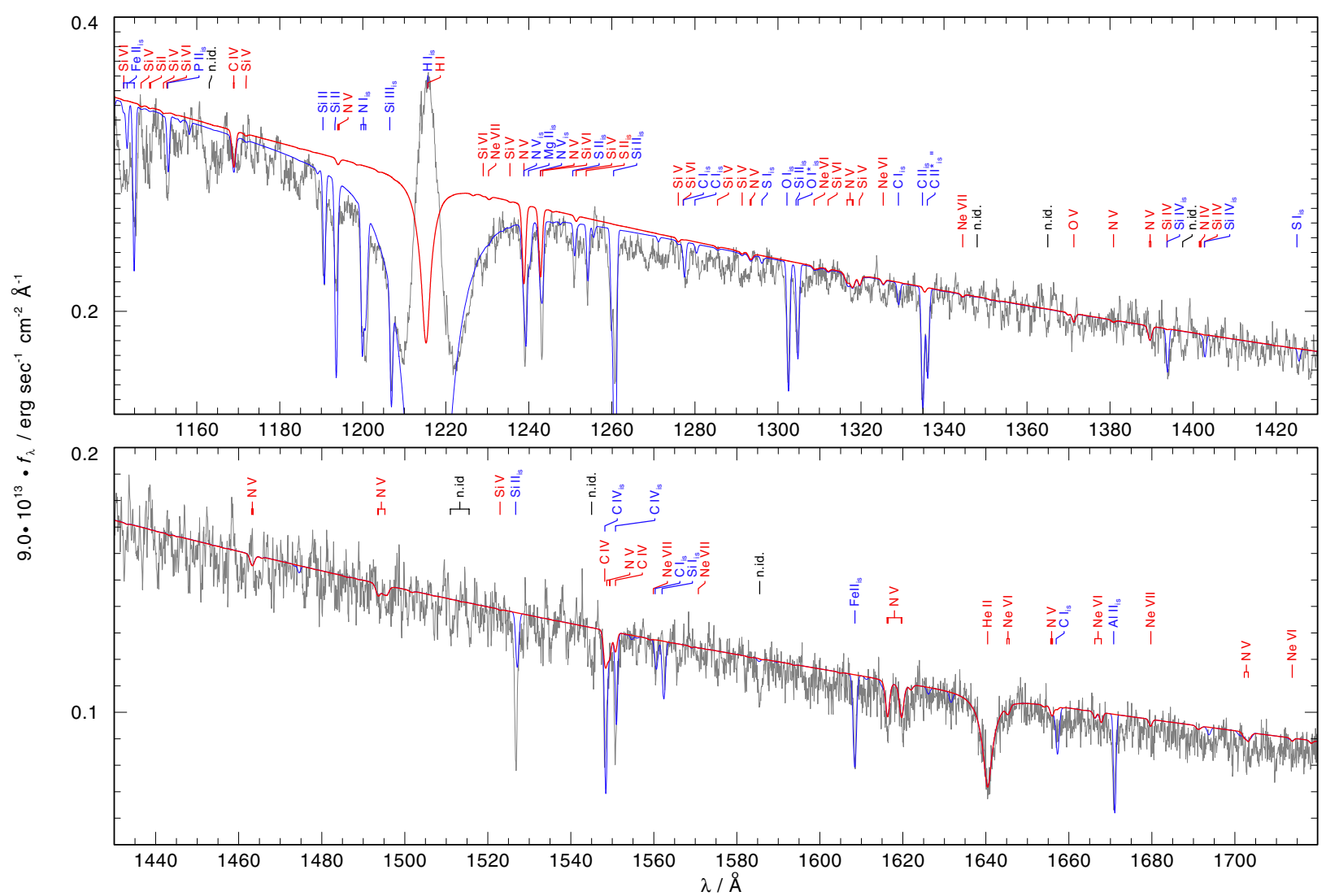

Fig. 5. HST/COS spectrum of K 1-27 (gray) compared with final synthetic spectra (red: pure stellar, blue: combined stellar and interstellar). The locations of photospheric (red) and interstellar (blue) lines reproduced by the synthetic spectrum are marked.

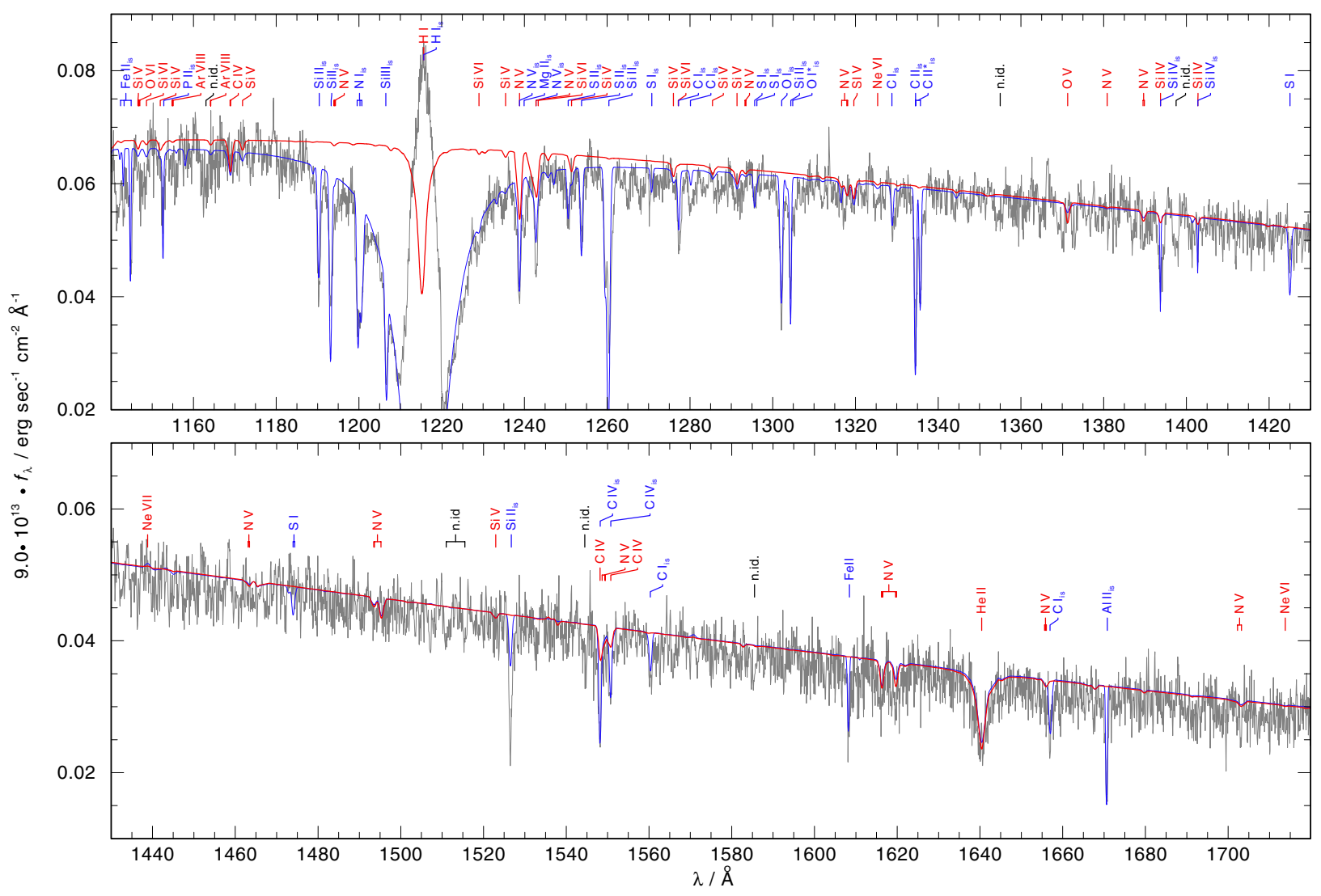

Fig. 6. Same as Fig. 5 for LoTr 4. 
A\&A 566, A116 (2014)

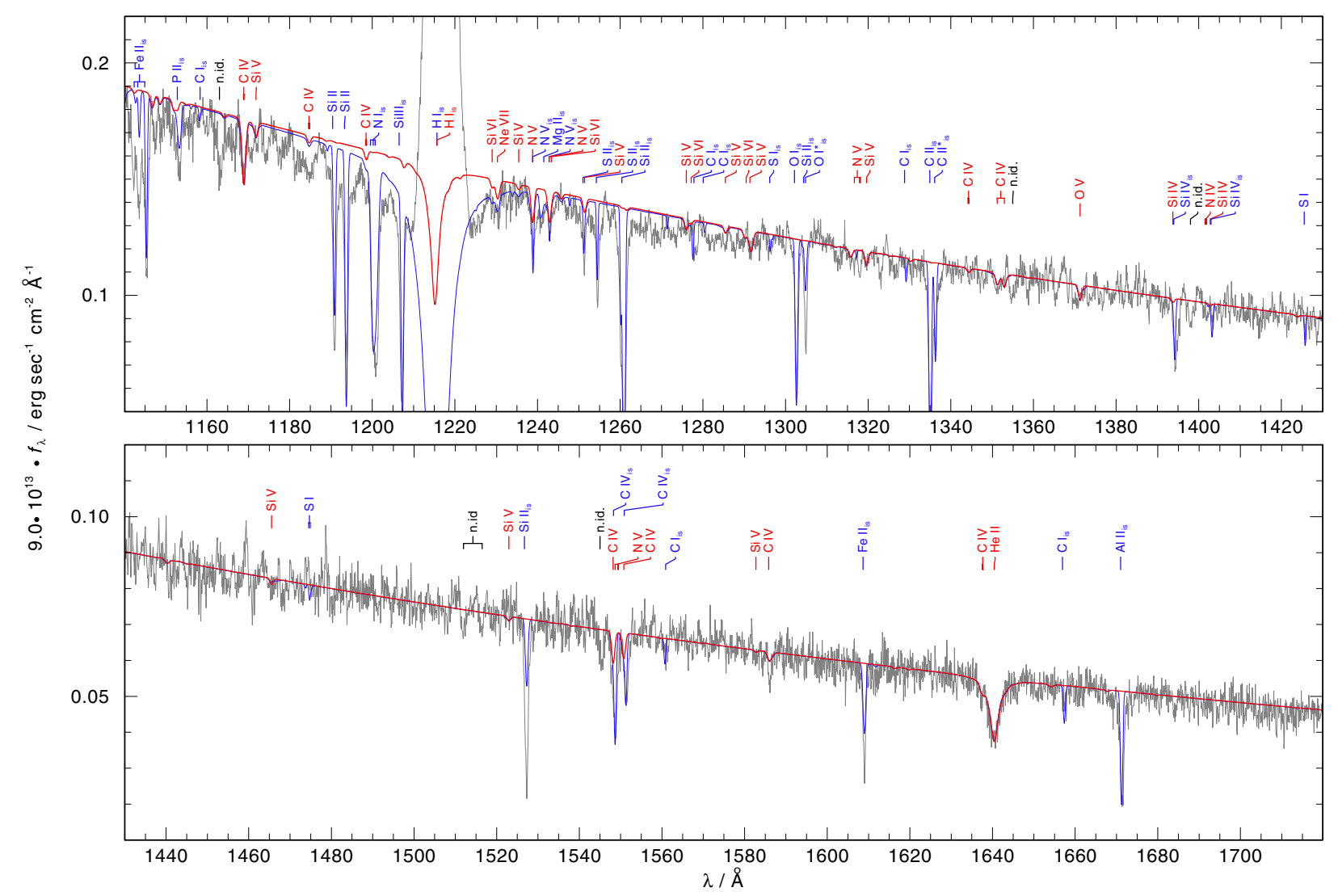

Fig. 7. Same as Fig. 5 for HS 1522+6615.

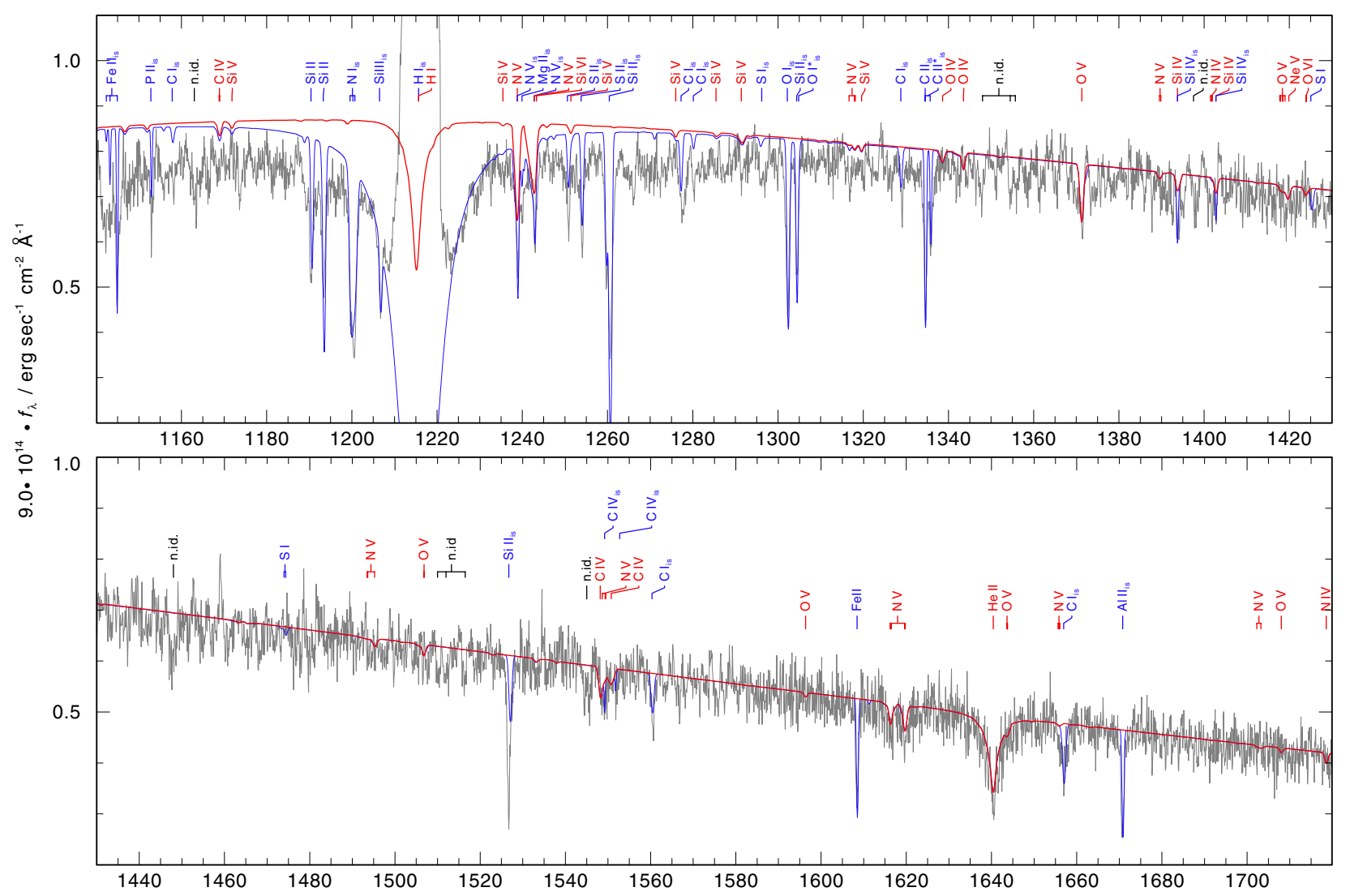

Fig. 8. Same as Fig. 5 for HS $2209+8229$. 
N. Reindl et al.: On helium-dominated stellar evolution: the mysterious role of the $\mathrm{O}(\mathrm{He})$-type stars
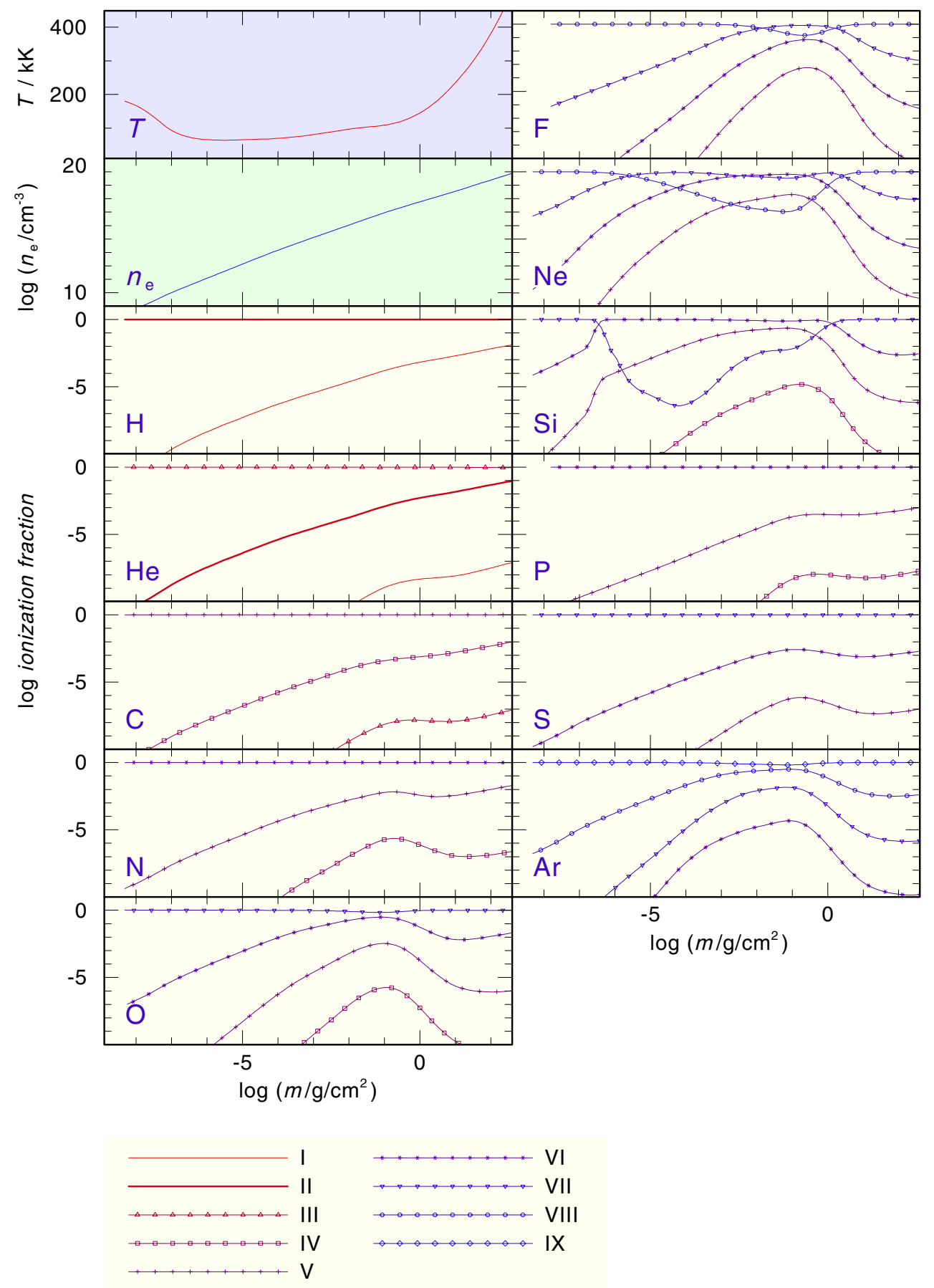

$T_{\text {eff }}=135 \mathrm{kK} \quad \log g=6.4$

Fig. 9. Temperature, electron density stratification, and ionization fractions of all elements in our final model of K 1-27. 
A\&A 566, A116 (2014)
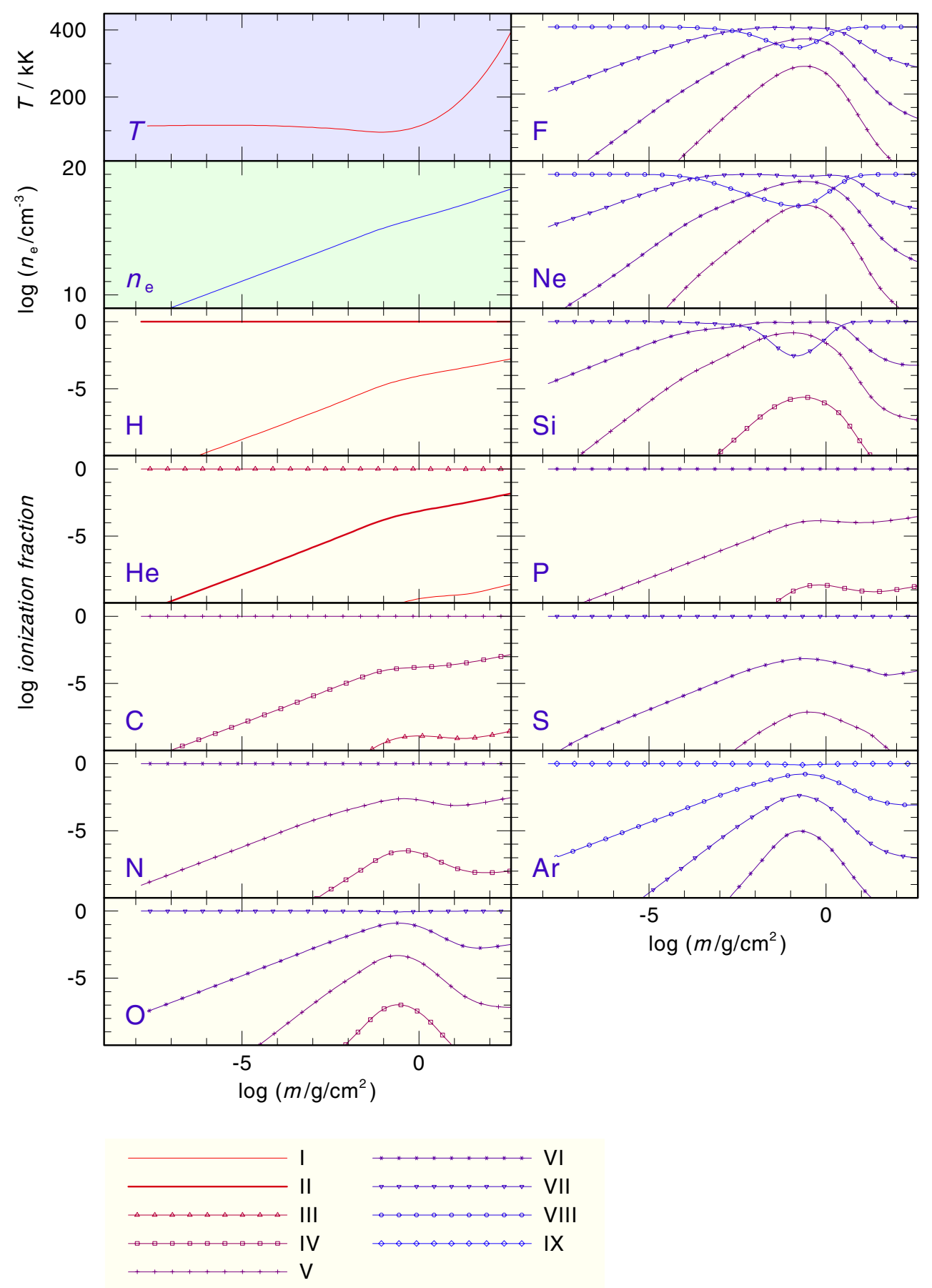

$T_{\text {eff }}=120 \mathrm{kK} \quad \log g=5.8$

Fig. 10. Like Fig. 9, for LoTr 4. 
N. Reindl et al.: On helium-dominated stellar evolution: the mysterious role of the $\mathrm{O}(\mathrm{He})$-type stars
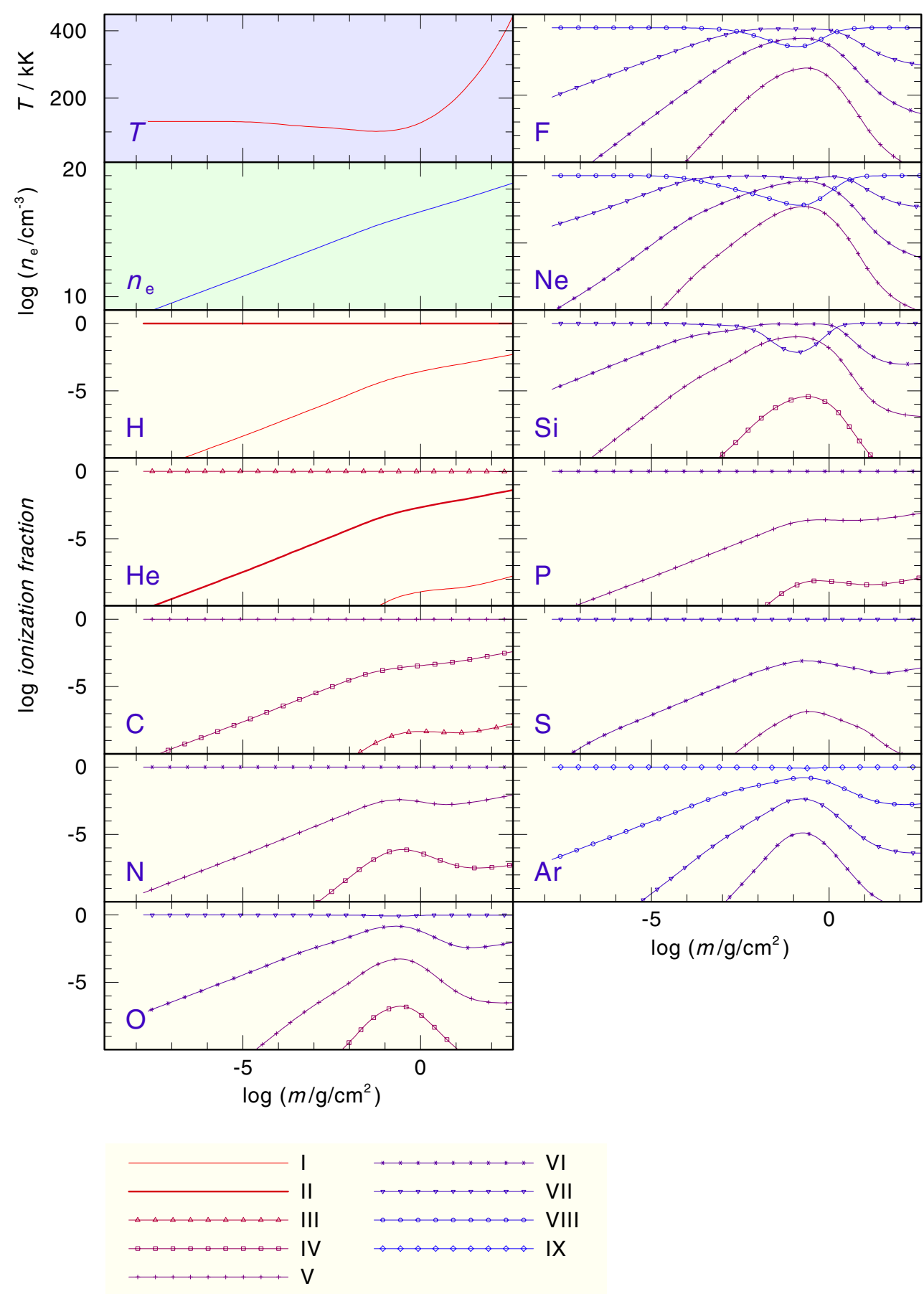

$T_{\text {eff }}=130 \mathrm{kK} \quad \log g=5.9$

Fig. 11. Like Fig. 9, for HS 1522+6615. 
A\&A 566, A116 (2014)
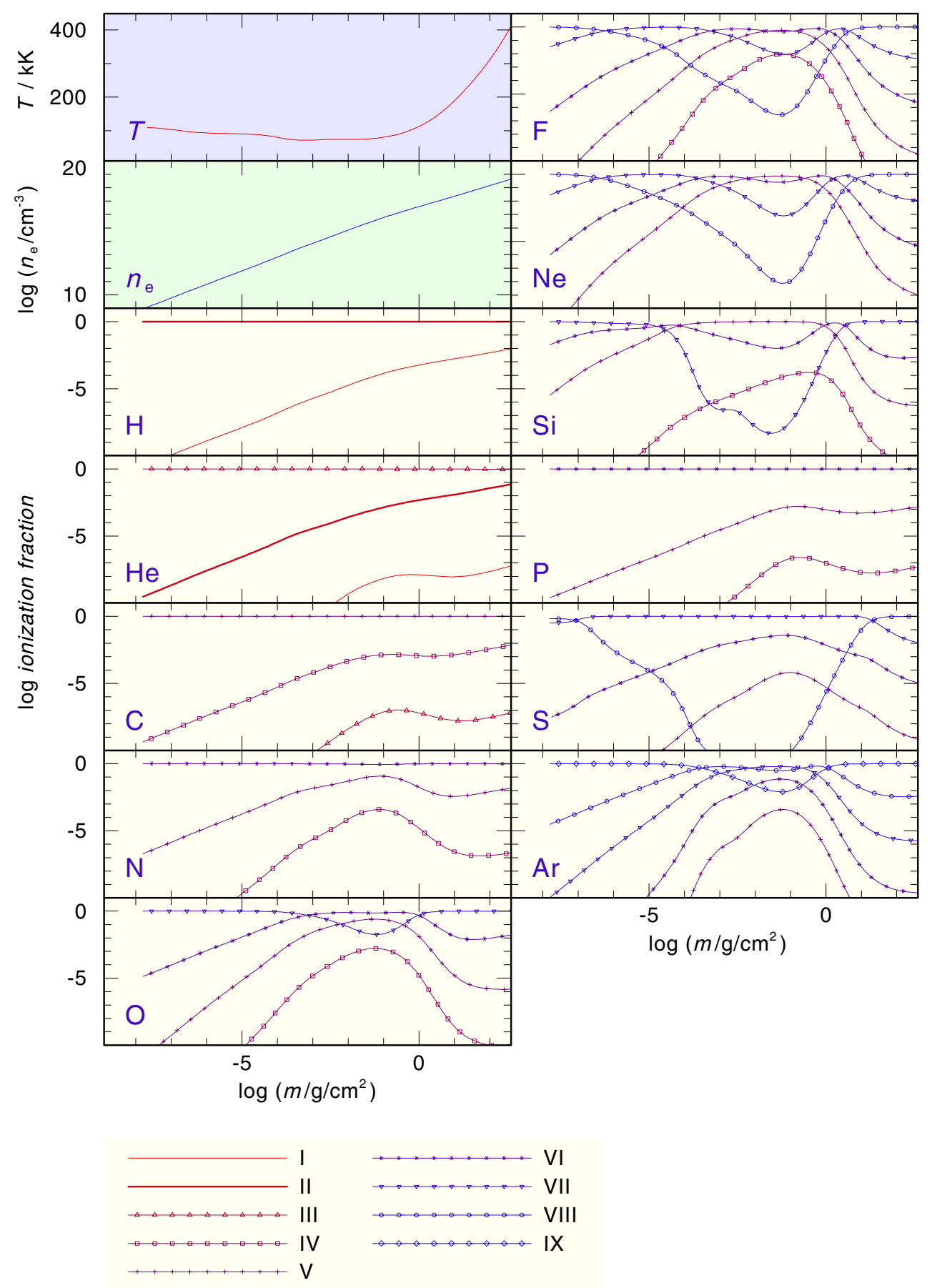

$T_{\text {eff }}=110 \mathrm{kK} \quad \log g=6.0$

Fig. 12. Like Fig. 9, for HS 2209+8229. 
N. Reindl et al.: On helium-dominated stellar evolution: the mysterious role of the $\mathrm{O}(\mathrm{He})$-type stars
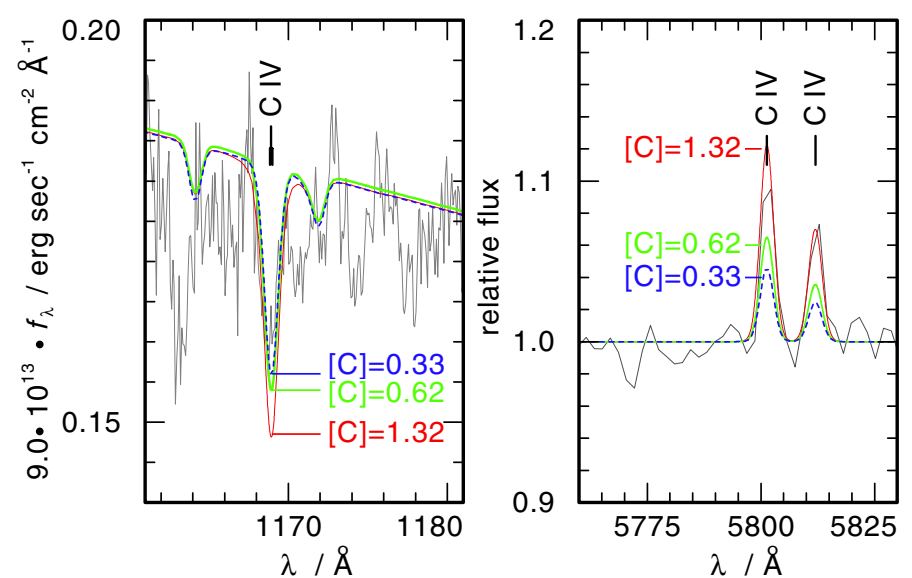

Fig. 21. Determination of the $\mathrm{C}$ abundance of HS $1522+6615$. C IV lines are compared with models with different $\mathrm{C}$ abundances as indicated by the labels.

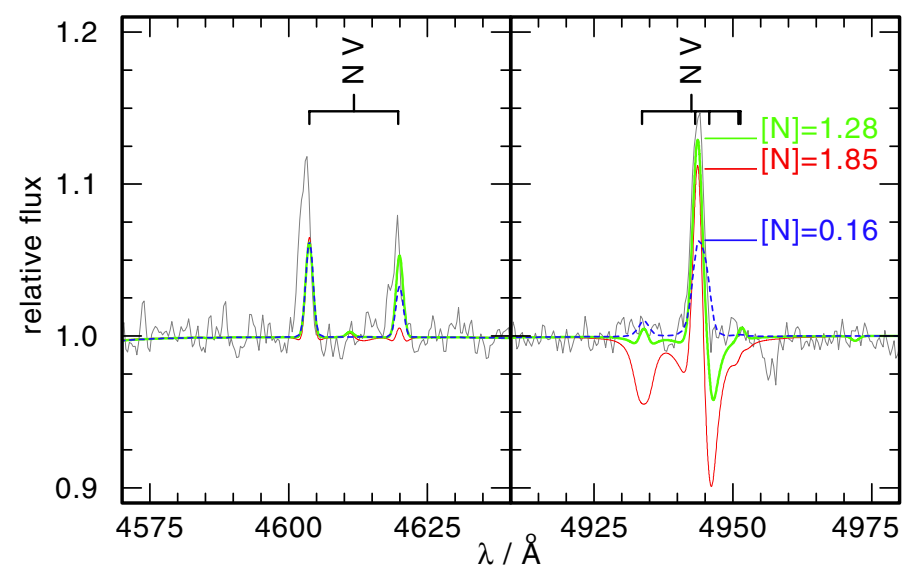

Fig. 22. Determination of the $\mathrm{N}$ abundance of K 1-27. N v lines in the EFOSC 2 observation are compared with models with different $\mathrm{N}$ abundances as indicated by the labels.

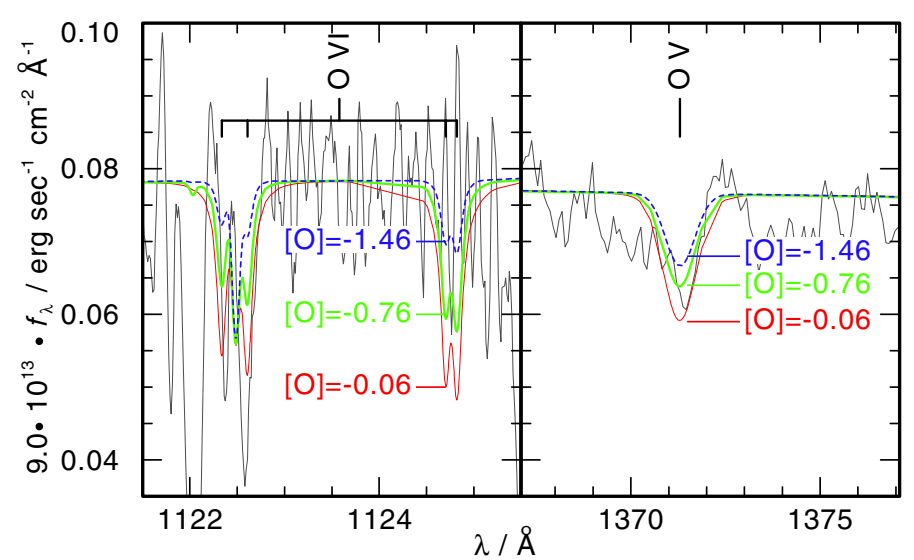

Fig. 23. Determination of the O abundance of HS 2209+8229. O V and $\mathrm{O}$ VI lines are compared with models with different $\mathrm{O}$ abundances as indicated by the labels.

Table 1. Statistics of the model atoms used in our TMAP calculations.

\begin{tabular}{|c|c|c|c|c|c|c|}
\hline \multirow[b]{3}{*}{ ion } & \multicolumn{6}{|c|}{$T_{\text {eff }}$} \\
\hline & \multicolumn{3}{|c|}{$>110 \mathrm{kK}$} & \multicolumn{3}{|c|}{$\leq 110 \mathrm{kK}$} \\
\hline & NLTE & LTE & lines & NLTE & LTE & lines \\
\hline $\mathrm{HI}$ & 10 & 6 & 45 & 10 & 6 & 45 \\
\hline H II & 1 & - & - & 1 & - & - \\
\hline He I & 5 & 98 & 3 & 5 & 98 & 3 \\
\hline He II & 14 & 18 & 91 & 14 & 18 & 91 \\
\hline He III & 1 & - & - & 1 & - & - \\
\hline C III & 13 & 54 & 32 & 13 & 54 & 32 \\
\hline C IV & 14 & 44 & 35 & 14 & 44 & 35 \\
\hline $\mathrm{CV}$ & 1 & 0 & 0 & 1 & 0 & 0 \\
\hline N III & 1 & 65 & 0 & 1 & 65 & 0 \\
\hline N IV & 16 & 78 & 30 & 16 & 78 & 30 \\
\hline $\mathrm{NV}$ & 14 & 48 & 35 & 14 & 48 & 35 \\
\hline N VI & 1 & 0 & 0 & 1 & 0 & 0 \\
\hline OIV & 18 & 76 & 39 & 18 & 76 & 39 \\
\hline $\mathrm{OV}$ & 17 & 109 & 35 & 17 & 109 & 35 \\
\hline O VI & 14 & 48 & 33 & 14 & 48 & 33 \\
\hline O VII & 1 & 0 & 0 & 1 & 0 & 0 \\
\hline Fv & 1 & 10 & 0 & 1 & 10 & 0 \\
\hline F VI & 6 & 6 & 0 & 6 & 6 & 0 \\
\hline F VII & 2 & 4 & 0 & 2 & 4 & 0 \\
\hline F VIII & 1 & 0 & 0 & 1 & 0 & 0 \\
\hline Ne IV & 0 & 0 & 0 & 2 & 39 & 0 \\
\hline $\mathrm{NeV}$ & 14 & 80 & 18 & 14 & 80 & 18 \\
\hline $\mathrm{Ne}$ VI & 14 & 17 & 30 & 14 & 17 & 30 \\
\hline Ne VII & 15 & 94 & 27 & 15 & 94 & 27 \\
\hline Ne VIII & 14 & 90 & 35 & 1 & 0 & 0 \\
\hline Ne IX & 1 & 0 & 0 & 1 & 0 & 0 \\
\hline Si IV & 12 & 11 & 24 & 12 & 11 & 24 \\
\hline $\mathrm{SiV}$ & 25 & 0 & 59 & 25 & 0 & 59 \\
\hline Si VI & 45 & 195 & 193 & 45 & 195 & 193 \\
\hline Si VII & 1 & 0 & 0 & 1 & 0 & 0 \\
\hline PIV & 15 & 36 & 9 & 15 & 36 & 9 \\
\hline $\mathrm{PV}$ & 18 & 7 & 12 & 18 & 7 & 12 \\
\hline P VI & 1 & 0 & 0 & 1 & 0 & 0 \\
\hline S v & 23 & 87 & 47 & 23 & 87 & 47 \\
\hline S VI & 25 & 12 & 25 & 25 & 12 & 25 \\
\hline S VII & 1 & 0 & 0 & 1 & 0 & 0 \\
\hline Ar V & & & & 1 & 359 & 0 \\
\hline Ar VI & 1 & 183 & 0 & 14 & 170 & 16 \\
\hline Ar VII & 40 & 112 & 130 & 40 & 112 & 130 \\
\hline Ar VIII & 13 & 28 & 24 & 13 & 28 & 24 \\
\hline Ar IX & 1 & 0 & 0 & 1 & 0 & 0 \\
\hline
\end{tabular}

